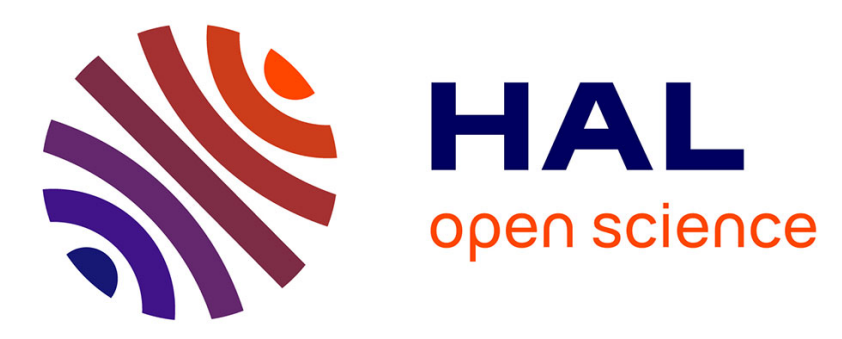

\title{
Artificial evolution of the morphology and kinematics in a flapping-wing mini UAV
}

\author{
Emmanuel De Margerie, Jean-Baptiste Mouret, Stephane Doncieux, \\ Jean-Arcady Meyer
}

\section{> To cite this version:}

Emmanuel De Margerie, Jean-Baptiste Mouret, Stephane Doncieux, Jean-Arcady Meyer. Artificial evolution of the morphology and kinematics in a flapping-wing mini UAV. Bioinspiration and Biomimetics, 2007, 2 (4), pp.65-82. 10.1088/1748-3182/2/4/002 . hal-02986347

\section{HAL Id: hal-02986347 https://hal.science/hal-02986347}

Submitted on 9 Nov 2020

HAL is a multi-disciplinary open access archive for the deposit and dissemination of scientific research documents, whether they are published or not. The documents may come from teaching and research institutions in France or abroad, or from public or private research centers.
L'archive ouverte pluridisciplinaire HAL, est destinée au dépôt et à la diffusion de documents scientifiques de niveau recherche, publiés ou non, émanant des établissements d'enseignement et de recherche français ou étrangers, des laboratoires publics ou privés. 


\title{
Artificial evolution of the morphology and kinematics in a flapping-wing mini UAV.
}

\author{
E. de Margerie, J.B. Mouret, S. Doncieux and J.-A. Meyer \\ SIMA dpt, ISIR, Université Pierre et Marie Curie \\ 104 av. Pdt Kennedy, 75016 Paris, FRANCE. \\ corresponding author: edemargerie@gmail.com
}

\begin{abstract}
Birds demontrate that Flapping-wing flight (FWF) is a versatile flight mode, compatible with hovering, forward flight, and gliding to save energy. This extended flight domain would be especially useful on mini UAVs. However, design is challenging because aerodynamic efficiency is conditionned by complex movements of the wings, and because many interactions exist between morphological (wing area, aspect ratio) and kinematic parameters (flapping frequency, stroke amplitude, wing unfolding). Here we used Artificial Evolution to optimise these morpho-kinematic features on a simulated $1 \mathrm{~kg}$ UAV, equipped with wings articulated at the shoulder and wrist. Flight tests were conducted in a dedicated steady aerodynamics simulator. Parameters generating horizontal flight for minimal mechanical power were retained. Results showed that flight at medium speed $(10-12 \mathrm{~m} / \mathrm{s})$ can be obtained for reasonnable mechanical power $(20 \mathrm{~W} / \mathrm{kg})$, while flight at higher speed $(16-20 \mathrm{~m} / \mathrm{s})$ implied increased power $(30-50 \mathrm{~W} / \mathrm{kg})$. Flight at low speed $(6-8 \mathrm{~m} / \mathrm{s})$ necessitated unrealistic power levels (70-500 W/kg), probably because our simulator neglected unsteady aerodynamics. The underlying adaptation of morphology and kinematics to varying flight speed were compared to available biological data on the flight of birds.
\end{abstract}




\section{Introduction}

In order to achieve sustained horizontal flight, human flying machines usually rely on a fixed wing and a powered propeller such as an airplane, or on powered rotating wings like in helicopters. On the other hand, birds, bats, and insects - i.e., actively flying animals - use flapping-wing flight (FWF) to produce the lift and thrust forces needed for forward flight. The reasons why biological and technical worlds have retained different solutions may be of both historical (e.g., contingency) and structural (e.g., material constraints) nature. In particular, it appears that:

- Continually, rotating mechanical joints - on which propellers and rotors are based in human technology - do not exist in animals at the macroscopic, morphological level. Skeletal joints would belong to the category of rotating joints, but the dependence on muscles and tendons as force and torque effectors limit their angular rotation. Hence only reciprocating movements between skeleton elements are possible in animals. As a consequence of the historical, contingent constraint of inheriting a muscle-based activation system, propellers or rotating wings for active flight are beyond the reachable phenotypes of extant animals.

- Articulated, moving wings as necessitated by FWF are hard to design for human flight. At a man-lifting scale, with usual aeronautical materials such as wood, steel, aluminium or even newer composite materials, flapping-wing flight is a tremendous aerodynamic, mechanical and structural challenge for current technology. Although several "ornithopters" have been constructed in the last 100 years, even the most recent designs (DeLaurier 1999) remain marginally efficient compared to classical fixed-wing or rotor designs. As a result, the great potential of FWF demonstrated by animals in terms of speed range or manoeuvrability, though attractive, remains beyond the achievable goals of today's human aerial transportation prospect.

Although FWF seems rather impractical at a man-lifting scale, which is also illustrated by the fact that flying animals rarely exceed $10 \mathrm{~kg}$ in mass, the recent technological field of unmanned aerial vehicles (UAVs) may find in FWF solutions to challenging flight dynamics problems. As small size is a determining factor, research efforts on FWF are mostly focused on micro-UAVs (insect to small bird-sized: $1-100 \mathrm{~g}$ ) and mini-UAVs (medium to large bird-sized: $0.1-10 \mathrm{~kg}$ ). At these sizes, FWF has the potential to allow unique flight dynamics abilities, as demonstrated by the performance of flying animals :

- FWF is versatile. Depending on specific size and weight, flying animals can hover, fly forward at varying speeds, and glide or soar to save energy. Many species can perform all three flight regimes, as exemplified by the European kestrel, F. tinnunculus. These regimes are selected according to daily activities like foraging, observation or migration. These species merge both helicopter and airplane-like abilities into a single, extended flight domain.

- Active articulated wings and asymmetrical flapping provide very high manoeuvrability, especially useful in obstructed spaces, as demonstrated by perching birds that fly among tree branches for example.

- Reciprocating wing movements allow flying animals to use favourable unsteady aerodynamics, at least during hovering and slow flight (Norberg 1990). For example, one well-known unsteady effects is the "delayed stall", which can increase the airfoil maximal lift up to 50\% when the wing's angle of attack is suddenly increased (Vogel 1994).

Drawing inspiration from natural flyers, one of the main interests in transposing FWF to small UAVs is the ability to obtain an extended flight-mode range. Namely, a hovering and very slow 
flight appears useful for exploration and observation in obstructed or urban areas, while a medium to high speed horizontal flight at low energetic cost, which is not well achieved by helicopters, is necessary to cover large distances. Moreover, the ability to soar in ascending air currents is a supplementary key-feature for energy saving.

Although the perspective of implementing these three flight modes on a single UAV is very attractive, the main drawback in using FWF, as mentioned above, is the high difficulty of designing a mechanically and aerodynamically functional flapping wing, because of the large number of interacting parameters like morphological characteristics, degrees of freedom and kinematic data associated with the wing's parts.

In an attempt to overstep these difficulties, we used Artificial Evolution (AE) to explore the range of functional wing morphologies and kinematics for a simulated bird-like mini UAV. AE is a "trial and error" optimisation method inspired from Darwinian natural selection. It may call upon several numerical optimisation procedures such as "Genetic Algorithms", "Evolution Strategies", and others (Goldberg 1989), which are used in engineering, artificial intelligence and biology to solve complex problems (for an AE application to biological morphology, see de Margerie et al. 2005). Compared to classical knowledge-driven engineering methods, AE has the main advantage that it does not need a comprehensive high-level knowledge of the considered problem. In our case, only the basic laws of aerodynamics need to be implemented in a simulator, to make the generation of various FWF solutions possible. By selecting the best among such randomly generated solutions, by randomly recombining and mutating them, and by testing the "offspring" solutions again, AE can generate satisfactory optimised solutions to intricate problems, with minimal initial knowledge. This can be useful for solving the FWF problem because one only needs to know rather general aerodynamic laws to test any AE-produced wing morphology, or any flapping movement, in a flight simulator.

The present work is part of the ROBUR project (Doncieux et al. 2004) that aims at designing an outdoor bird-like mini UAV of 1-2m wingspan, with adaptive locomotion modes and abilities required for a true decisional autonomy like obstacle avoidance (Muratet et al. 2005), localization and mapping (Angeli et al. 2006) and energy management (Barate et al. 2006) . Here we focus on wing morphology and movements and use $\mathrm{AE}$ to find morphological and kinematic parameters providing flapping flight at minimal energetic cost. These parameters mainly include the wing area, the wing aspect ratio, the flapping frequency, the stroke amplitude and the angles of attack. As a first stepping stone, before later considering turning, ascending or aerobatic flight, we concentrated the present work on forward horizontal flight at varying speeds $\left(6-20 \mathrm{~m}_{\mathrm{s}} \mathrm{s}^{-1}\right)$, for an approximately 1 $\mathrm{kg}$ bird-like UAV.

A few previous works have used AE to optimize FWF, but with notable differences relatively to the present work. Salles and Schiele (2004), for instance, optimised the movement of a small rigid wing inspired from an hawkmoth's wing, manipulated at low Reynolds number by a robotic arm, using a genetic algorithm. Van Breugel and Lipson (2005) used an evolutionary algorithm to optimise the lift produced by a simulated 50-310g four-wing ornithopter. Although not using AE, Rakotomamonjy et al. (2004) used an optimisation algorithm (non linear programming) on a neural network controlling the kinematics of a simulated $30 \mathrm{~g}$ micro-UAV, in order to maximize the lift forces. Beyond differences in the UAV's mass, our work differs from these previous studies by the fact that we simultaneously optimize the kinematics and the wing morphology (size and shape) of our UAV. To our knowledge, the work of Shim et al. (2004) is the only other study evolving both the morphology and kinematics of bird- or bat-sized FWF UAVs. However, their optimisation process does not consider energy consumption - their fitness criterion being a sum of flight speed and hovering time - and accordingly embeds their study in a different perspective tied to artificial life and virtual worlds and then less realistic and applicable to real UAVs than ours. 


\section{Methods}

\subsection{UAV morphology and kinematics}

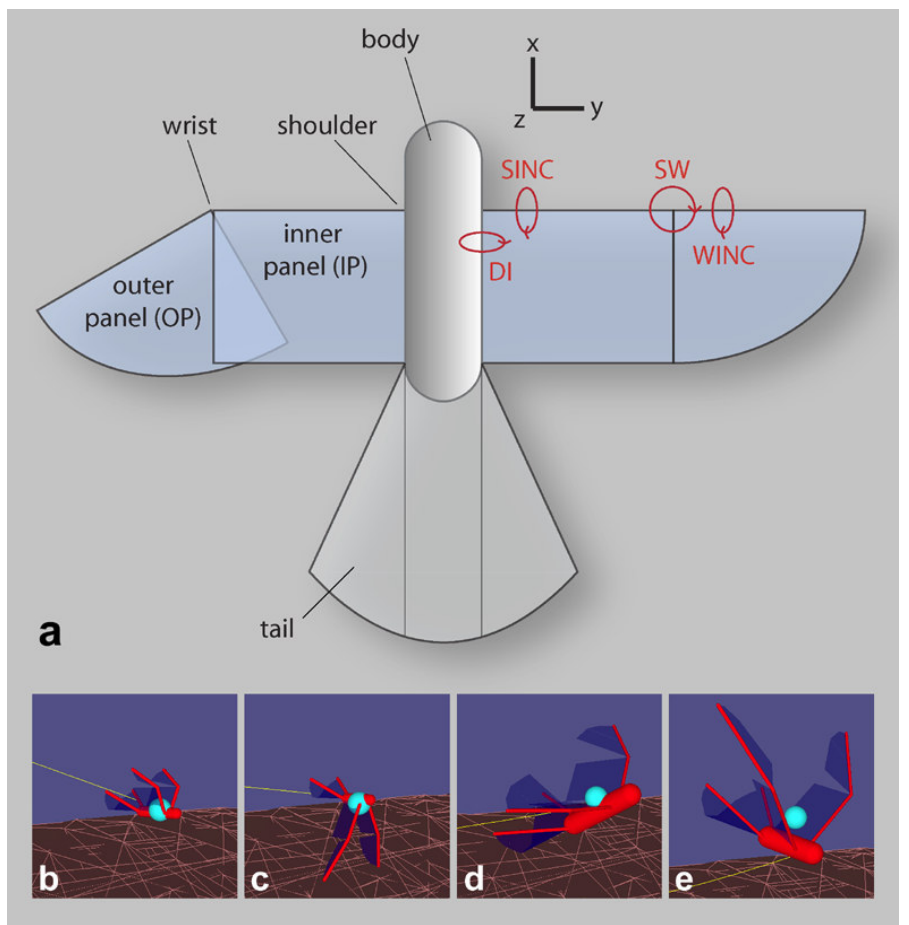

Figure 1 : UAV Morphology. a: Wing panels and their degrees of freedom (DOFs) ; dihedral (DI), sweep (SW), shoulder incidence (SINC) and wrist incidence (WINC). b, c, d, e: four possible morphologies, for extreme values of the wing area $\left(a_{w}\right)$ and the wing aspect ratio $\left(\lambda_{w}\right)$. UAVs are displayed in flight, with random angular values on the four DOFs. The lightblue sphere indicates the position of the UAV's center of gravity. The yellow line is the trajectory of the UAV's body. $\mathbf{b}$ : $\mathrm{a}_{w}$ $=0.1 \mathrm{~m}^{2}, \lambda_{\mathrm{w}}=4.5$, wingspan $=0.67 \mathrm{~m} . \mathbf{c}: \mathrm{a}_{\mathrm{w}}=0.1 \mathrm{~m}^{2}, \lambda_{\mathrm{w}}=10$, wingspan $=1 \mathrm{~m} . \mathbf{d}: \mathrm{a}_{\mathrm{w}}=0.4 \mathrm{~m}^{2}, \lambda_{\mathrm{w}}=4.5$, wingspan $=1.34$ m. e: $a_{w}=0.4 \mathrm{~m}^{2}, \lambda_{w}=10$, wingspan $=2 \mathrm{~m}$.

Freely inspired from bird morphology, our simulated UAV had two symmetrical wings and a central tail, as described on fig.1. Each wing comprised an inner rectangular and an outer elliptic panel of equal spans, named IP and OP, respectively. Each wing had 4 degrees of freedom (DOF): rotation was possible in dihedral (x) and incidence (y) at the "shoulder" joint, i.e. between body and IP, while rotations in sweep (z) and incidence (y) were allowed at the "wrist" joint, i.e. between IP and OP. Rotation in sweep allowed the UAV to possibly retract its wings during the flapping stroke. A sweep angle implied that IP and OP partly overlap, hence entailing a decrease in the aerodynamically efficient wing area. This overlap interacted freely with incidence rotation at the wrist, i.e. potential collisions between panels were avoided by continuously modifying the panels' shape at their joint. As dihedral movements only happened at the shoulder and sweep movements at the wrist, these two DOFs will be referred to as "dihedral" (DI) and "sweep" (SW). Likewise, the two incidence DOFs will be distinguished with the terms "shoulder incidence" (SINC) and "wrist incidence" (WINC).

The size, shape and movements of the wings were determined by 12 parameters which were allowed to vary - within the bracketed limits given below - during the AE optimisation process. These parameters constituted the "genome" of our UAV:

1 - The wing area $\left(a_{w}\right)\left[0.1-0.4 m^{2}\right]$ was the "size" parameter, i.e. the sum of areas of both wings fully extended (no sweep). 
2 - The wing aspect ratio $\left(\lambda_{\mathrm{w}}\right)[4.5-10]$ was the "shape" parameter, expressing the ratio of wing span - both wings included - to wing chord.

3 - The flapping frequency (f) $[1-10 \mathrm{~Hz}]$ was common to all DOFs - shoulder dihedral and incidence, wrist sweep and incidence - which were all controlled through sinusoidal functions.

4 - The dihedral amplitude $\left(\mathrm{amp}_{\mathrm{di}}\right)\left[0-89^{\circ}\right]$ was the amount of $\mathrm{x}$ angular oscillation between body and wing. Upward and downward rotation ranges of the wings were symmetric (e.g. $89^{\circ}$ upward and $89^{\circ}$ downward, zero-centered). The max value of $89^{\circ}$ was chosen to prevent geometrical and numerical singularities at $90^{\circ}$.

5 - The sweep amplitude $\left(\mathrm{amp}_{\mathrm{sw}}\right)\left[0-89^{\circ}\right]$ was the maximal $\mathrm{z}$ angle between IP and OP leading edges. Contrary to dihedral, this range holds only backward, i.e. OP could not rotate frontward to IP in a "negative sweep" configuration.

6 - The sweep offset $\left(\mathrm{off}_{\mathrm{sw}}\right)[0-500 \%$ of sinus period] determined the periodic offset between dihedral and sweep movements. A range of 500\%, instead of the theoretically sufficient $100 \%$, allowed this parameter to possibly evolve near to a $100 \%$ value without encountering any boundary.

7 - The shoulder incidence reference $\left(\right.$ ref $\left._{\text {sinc }}\right)\left[-20-20^{\circ}\right]$ is the default y angle between body and IP, around which the incidence sinusoidal oscillation occurred.

8 - The shoulder incidence amplitude $\left(\mathrm{amp}_{\mathrm{sinc}}\right)\left[0-69^{\circ}\right]$ is the amount of y angular oscillation between body and IP.

9 - The shoulder incidence offset $\left(\right.$ off $\left._{\text {sinc }}\right)[0-500 \%]$ is the periodic offset between dihedral and shoulder incidence movements.

10 - The wrist incidence reference $\left(\operatorname{ref}_{\text {winc }}\right)\left[-20-20^{\circ}\right]$ is the default y angle between IP and OP.

11 - The wrist incidence amplitude $\left(\mathrm{amp}_{\text {winc }}\right)\left[0-69^{\circ}\right]$ is the amount of y angular oscillation between IP and OP.

12 - The wrist incidence offset (off winc $_{\text {) }}[0-500 \%]$ is the periodic offset between dihedral and wrist incidence movements.

Based on the preceding parameters, the time-dependent kinematic laws of the angular variations in DOFs were:

$$
\begin{gathered}
D I=a m p_{d i} \cdot \sin (2 \cdot \pi \cdot f \cdot t) \\
S W=a m p_{s w} \cdot\left(\frac{1}{2}+\frac{1}{2} \cdot \sin \left(2 \cdot \pi \cdot\left(f . t+o f f_{s w}\right)\right)\right) \\
S I N C=r e f_{\text {sinc }}+a m p_{\text {sinc }} \cdot \sin \left(2 \cdot \pi \cdot\left(f . t+o f f_{\text {sinc }}\right)\right) \\
W I N C=r e f_{\text {winc }}+a m p_{\text {winc }} \cdot \sin \left(2 \cdot \pi \cdot\left(f . t+o f f_{\text {winc }}\right)\right)
\end{gathered}
$$

with $\mathrm{t}$ being the simulated time in seconds. 
The remaining features of the UAV may be inferred either from the evolving parameters just mentioned, or from deliberate constraints such as:

The span $\left(b_{w}\right)$ and inner chord $\left(c_{w}\right)$ of each wing were direct geometrical outcomes of the wing area and aspect ratio:

$$
\begin{gathered}
b_{w}=\frac{\sqrt{\lambda_{w} \cdot a_{w}}}{2} \\
c_{w}=\frac{a_{w}}{b_{w} \cdot\left(1+\frac{\pi}{4}\right)}
\end{gathered}
$$

The body of the UAV was a cylinder with rounded tips (fig.1). Its length $\left(l_{b}\right)$ was proportional to the wing chord, and its radius $\left(\mathrm{r}_{\mathrm{b}}\right)$ was a function of the wing area, such that the body cross-section was proportional to the wing area:

$$
\begin{aligned}
& l_{b}=\frac{4}{3} \cdot c_{w} \\
& r_{b}=\frac{\sqrt{a_{w}}}{10}
\end{aligned}
$$

The tail area $\left(\mathrm{a}_{\mathrm{t}}\right)$ was proportional to the wing area. The tail parts extending laterally beyond body sides were raised at $45^{\circ}$ around $\mathrm{x}$ axis to provide some lateral stability to the UAV through a Vshaped tail surface:

$$
a_{t}=\frac{a_{w}}{2}
$$

Tail position relative to the body was not allowed to change and remained constant along all experiments. A control of the tail should further increase our artificial bird stability, but we preferred not to include one, to rely on passive stability as much as possible.

The masses of the UAV elements were determined as follows:

Considering that the wing mass would represent a significant part of the total mass in a real FWF $\mathrm{UAV}$, and considering that we aim at designing an approximately $1 \mathrm{~kg}$ flyer, the body mass $\left(\mathrm{m}_{\mathrm{b}}\right)$ was set to:

$$
m_{b}=0.5 \mathrm{~kg}
$$

The wing mass $\left(\mathrm{m}_{\mathrm{w}}\right.$, for both wings) depended on the wing area through an isometric relationship inspired from Greenewalt (1975). The tail mass $\left(\mathrm{m}_{\mathrm{t}}\right)$ was estimated through a similar relationship:

$$
\begin{gathered}
m_{w}=2 .\left(a_{w}\right)^{1.5} \\
m_{t}=\left(a_{t}\right)^{1.5}
\end{gathered}
$$


The masses of wings and tail were concentrated in their respective leading edges, and uniformly distributed along the span. The mass distribution within the body was such that the whole UAV's center of gravity (CG) was located at about $25 \%$ of wing chord when the wings were fully extended.

The chosen ranges and laws of variation of our parameters can now be compared to biological data. Starting from the hard constraint that the body of our UAV had a $0.5 \mathrm{~kg}$ mass, we found several species with similar masses in the data of Magnan (1922, in Greenewalt, 1962). These species, among which are Hooded Crow (Corvus cornix), Shearwater (Calonectris diomedea) or MarshHarrier (Circus aeruginosus), have wing areas ranging from 0.13 to $0.23 \mathrm{~m}^{2}$, except some "duckmodel" fast-flying species like Shoveler (Anas clypeata) or Coot (Fulica atra), which have wing areas as low as $0.06 \mathrm{~m}^{2}$. Choosing a range of $(0.1-0.4)$ for $\mathrm{a}_{\mathrm{w}}$ covered the whole biological size range with the same mass, except the smallest, highly loaded duck-styled flyers, which can be considered as high-speed flight specialists, differing from our versatile UAV objective. As a direct outcome of the wing area, the wing loading of our UAV (ratio of total mass to wing area) could range from 2.6 for maximal $\mathrm{a}_{\mathrm{w}}$ to $5.7 \mathrm{~kg} . \mathrm{m}^{-2}$ for minimal $\mathrm{a}_{\mathrm{w} .}$. According to Greenewalt $(1975, \mathrm{p} .16)$, this range covers the natural wing loadings of all sampled groups, including raptors

[Falconiformes], owls [Strigiformes], herons [Ardeidae] and bats of comparable masses, except the highly loaded ducks and shorebirds. In the latter groups, wing loading usually attains $6-10 \mathrm{~kg} \cdot \mathrm{m}^{-2}$, while Auks [Alcidae] can attain even higher wing loadings, up to $24 \mathrm{~kg} \cdot \mathrm{m}^{-2}$.

Concerning wing masses, relationship (11) was inspired from isometric biological data, but yielded slightly higher UAV wing masses compared to the above mentioned bird species, especially at higher wing areas. For example, we predicted $\mathrm{m}_{\mathrm{w}}=0.22 \mathrm{~kg}$ for a $0.23 \mathrm{~m}^{2}$ wing area, whereas wings of a Marsh-Harrier are $0.14 \mathrm{~kg}$ according to Magnan. We chose to be rather conservative regarding this issue, as real articulated UAV wings will probably not equal natural performances regarding weight saving. We were also conservative when assuming a uniform wing mass distribution along the span of our UAV, rather than a decrease in mass toward the wing tips as it is observed in birds, which reduces the inertial power required for flapping flight (Van den Berg and Rayner 1995). According to Norberg (1990, p.173), the aspect ratio of birds weighting $0.75 \mathrm{~kg}$ - a rough estimation of the mean total mass of our UAV including body, wing and tail - averages 7.7. When individual species are considered in Magnan's data, $\lambda$ ranges from 6 for Hooded Crow to 9 for Shearwater. We allowed AE to search the optimal $\lambda$ between 4.5 and 10 . The lower boundary is imposed by our wing geometry for consistent panel overlap. The upper boundary at 10 was chosen to avoid generating high- $\lambda$ virtual morphologies that would probably not be stiff or strong enough in reality. This precaution was necessary, as our mechanical model did not take structural resistance into account (see below).

Last, the range of frequency we allowed $(1-10 \mathrm{~Hz})$ is similar to biological data corresponding to the same mass (2-10Hz; Norberg 1990 p.177).

Drawing inspiration from birds for setting the variation ranges of the morphology and kinematics of our UAV is deliberate. It does not warrant that the optimal FWF UAV will be found within these limits. It is definitely possible that a more efficient UAV design virtually exists beyond what nature has ever explored (especially using artificial construction materials). However as one observes natural flyers' skills and versatility, it is obvious that an UAV exhibiting at least some of these features would already be a large step forward compared to current humanly-designed flying machines. In a broader perspective, our deliberately bio-mimetic strategy falls within the scope of the "animat approach", which aims at designing simulated animals or real robots whose structure and functionalities are inspired from current biological knowledge, in the hope that they will exhibit at least some of the versatile capacities of real animals. Rather than direct inspiration consisting of copying the morphology and movements of a single bird species, here we launch artificial evolution within a biologically-informed search space, in order to obtain results that can be compared $a$ 
posteriori to biological data for validation and analysis, e.g. evolutionary trends and comparative adaptations. Interestingly, some of the results obtained in simulation, in a fully controlled environment (contrary to real world experiments) may, in turn, appear useful to biologists to disentangle the complex biological adaptations such as those involved in FWF.

\subsection{Flight simulation}

To test the flight characteristics of the multiple FWF solutions generated by the AE algorithm in terms of wing size, shape and movements, a flight simulator using the air speed vector at local points of the wing and tail was needed to compute the generated aerodynamic forces. This vector was a composition of both the UAV speed and the speed induced by the wing stroke. Moreover, possibly high local angles of incidence and lateral drift due to sweep had to be accounted for. We used a model specifically designed for flapping articulated wings (FMFAW, Flight Mechanics for Flapping Articulated Wings), which has been described elsewhere (Druot 2004). This semiempirical, quasi-steady-aerodynamics model considered that a wing was divided in a number of rigid flat quadrangular wing elements (WEL). In the present work, we divided each wing's IP into 3 coplanar WELs, and each OP into 6 coplanar WELs. At each time step $(0.005 \mathrm{sec}$ in the present work) and for each WEL, the model estimated the local incident airspeed, and computed 3 cumulative aerodynamic forces: the leading edge lift, the parachute drag and the friction drag, as decribed in more details by Druot (2004) . As the size and shape of our wings could vary, it was necessary that the model took the aspect ratio and the Reynolds number into account. The wing aspect ratio $\left(\lambda_{\mathrm{w}}\right)$ was accounted for by classical induced lift and drag formulae:

$$
\begin{gathered}
C l_{\lambda}=C l \cdot \frac{\lambda_{w}}{\lambda_{w}+2} \\
C d_{\lambda}=C d+\frac{C l^{2}}{\pi \cdot \lambda_{w}}
\end{gathered}
$$

with $\mathrm{Cl}_{\lambda}, \mathrm{Cd}_{\lambda}$ being the lift and drag coefficients corrected for aspect ratio, and $\mathrm{Cl}, \mathrm{Cd}$ uncorrected coefficients, i.e. at infinite aspect ratio. $\lambda_{w}$ was common to all WELs, such that the whole wing's aspect ratio affected the performance of each WEL, whereas the wing movement effects on the effective aspect ratio were neglected for simplicity. Moreover, no particular loss of aerodynamic efficiency was assumed at the body/IP and IP/OP interfaces because it was uneasy to determine $a$ priori what mechanical solution would be adopted for the real UAV's shoulder and wrist, and because we did not want the optimisation process to depend on such matters initially.

The Reynolds number $(\mathrm{Re})$ was assumed to have an effect on the friction drag coefficient in FMFAW $\left(\mathrm{Cd}_{\mathrm{f}}\right)$. According to Norberg (1990), we assumed a dependence on $\mathrm{Re}^{0.5}$ for laminar flow, and on $\operatorname{Re}^{0.2}$ for turbulent flow:

$$
C d_{f}=\max \left(8 \operatorname{Re}^{-0.5} ; 0.2 \operatorname{Re}^{-0.2}\right)
$$

Contrary to the aspect ratio, $\mathrm{Re}$ (and thus $\mathrm{Cd}_{\mathrm{f}}$ ) was considered as a "local" variable, computed at each time step and for each WEL individually.

As a result of (15), the transition from laminar to turbulent flow happened in our model around Re $2.10^{5}$. As for other parameters in FMFAW, e.g., those setting the dependence of the lift coefficient on the angle of incidence, proportionality coefficients in (15) were chosen to have the closest possible fit with experimental data for a particular airfoil. We chose the Selig 4083 airfoil, which is a $8 \%$ thick, under-cambered airfoil designed for providing high lift and lift/drag ratio at low Reynolds number $\left(6.10^{4}-2 \cdot 10^{5}\right)$. Its performances were measured experimentally in a wind tunnel 
for Re $6.10^{4}-3.10^{5}$ (Selig 1997). Figure 2 compares these experimental curves with the outcome of FMFAW as used in the present work. Beyond providing useful experimental data to calibrate our model, we chose the Se 4083 for its affinities with wing airfoils in birds, in terms of shape, thickness, camber, maximum lift coefficient and Reynolds number range (Withers 1981).
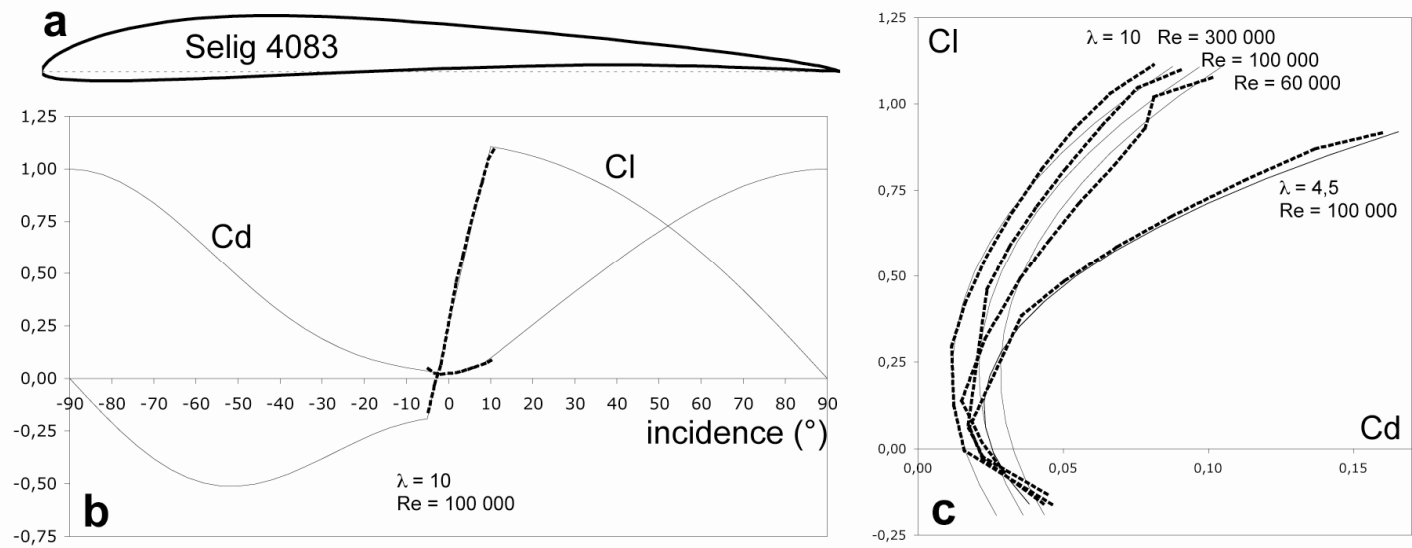

Figure 2 : Lift and drag coefficients as computed by the FMFAW model (thin lines), compared to experimental measures on the Selig 4083 airfoil (corrected for aspect ratio effect, dotted lines). a: outline of the Selig 4083 airfoil. b: $\mathrm{Lift}(\mathrm{Cl})$ and Drag $(\mathrm{Cd})$ coefficients of wing surface elements, as a function of angle of incidence. Although experimental data are only available in the "common" incidence range $\left(-5\right.$ to $\left.10^{\circ}\right)$, FMFAW estimates values for the whole $180^{\circ}$ incidence range. c: effects of Reynolds number $(R e)$ and aspect ratio $(\lambda)$ variations on the polar lift-drag curve.

Concerning the UAV tail, aerodynamic forces were calculated similarly to the wings, but assuming a symmetrical Naca 009 airfoil, and a 1.0 value for aspect ratio. Finally, the body of the UAV was assumed to produce drag only, with a drag coefficient of 0.3 indexed on its frontal area (Norberg 1990, p.165).

Although the fit between FMFAW aerodynamic forces and experimental data at varying Reynolds number seems rather satisfactory (fig.2), FMFAW remains based on steady aerodynamics, and thus does not compute unsteady aerodynamics effects, nor interactions between UAV's parts. Thus the quantitative results of simulations must be interpreted with enough caution, especially at low flight speed, where unsteady effects, interactions and flight in disturbed air grow in importance. For this reason, we optimised our UAV using FMFAW for flight speeds ranging from 6 to $20 \mathrm{~m} . \mathrm{s}^{-1}$, but not for lower flight speeds, nor for hovering flight.

Aside FMFAW and flight mechanics, we used the Open Dynamics Engine (ODE, Smith 2006) to simulate the relative movements of each part of our articulated UAV and compute its flight trajectory. Body and wing parts, considered as not deformable solids, were attached using joints having the same DOFs as in fig. 1. Sinusoidal angular movement of these 4 DOFS were obtained by producing enough torque at the joints to follow precisely the desired kinematic curve as dictated by the UAV's genome. High torques, possibly up to unrealistic values, were allowed to be produced at some joints if this was necessary to follow the "genetic" kinematics against possibly strong external forces, i.e. weight and aerodynamic forces on wing panels. It was the role of evolution to find an adequate, realistic wing morphology and movement that minimized the torques at joints, and hence decreased the required mechanical power, while achieving forward flight at a given speed.

\subsection{Evaluation, Fitness and Evolutionary Algorithm}

First, we briefly remind the general principles of Artificial Evolution, and how AE draws 
inspiration from natural random variation and Darwinian selection.

Each potential solution generated by AE is called an "individual", as its characters are dictated by a genome (a chain of 12 floating point numbers in the present work), and expressed into a phenotype (specific morphology and kinematics) interacting with a simulated world (flight simulation). The "fitness" of each individual relatively to the chosen problem (forward horizontal flight) is measured during its lifetime (the duration of an evaluation), and determines its breeding success, i.e. the chance that its genome will be selected for creating a new individual or "offspring" at the next evolution step. When an individual possesses a high fitness and is selected for offspring production, its genome is copied, crossed with the genome of another selected individual, randomly mutated, and then expressed into the offspring's phenotype, which is evaluated in turn.

In the present case, the fitness of each individual was assessed through a standardized test flight: the UAV was launched forward at an initial 300m height, with a given initial horizontal speed which was constant for all individuals within an evolutionary "run" (e.g. $10 \mathrm{~m} . \mathrm{s}^{-1}$ ). The genetically determined kinematics of the individual were symmetrically applied at joints of both wings since the first time step of evaluation and for 10 seconds - i.e. 2000 time steps - during which the UAV flew freely: no particular constraint was applied to its trajectory to "help" it achieve a stable horizontal flight. At each time step of the evaluation, two variables were recorded in order to quantify the fitness of the individual:

- the distance (D) between the UAV's body and the ideal "reference" trajectory, i.e. an horizontal path at the initial launch speed (e.g. $10 \mathrm{~m} \cdot \mathrm{s}^{-1}$ ).

- the instantaneous mechanical power $(\mathrm{P})$ produced at the wings' joints (shoulders and wrists). This variable was computed as the sum, for all 4 joints, of the scalar product between the instantaneous torque $(\tau)$ and the instantaneous rotational speed $(\omega)$.

$$
P=\sum_{i=1}^{4}\left|\vec{\tau}_{i} \cdot \vec{\omega}_{i}\right|
$$

with i referring to each individual joint.

Note that the power was counted positive regardless of the sign of the scalar product. This means that the torques produced to accelerate the instantaneous joint rotation and the torques used to slow down the rotation were assumed to have equivalent energetic costs. We chose this conservative hypothesis which maximized the power consumption, a priori assuming that the real UAV would probably not have an elastic energy storage capacity. Referring to assumptions in the biological literature about the power consumption of birds, some authors consider the acceleration only and ignore the decelerating power, although some other recommend to add both, as we did (Van den Berg and Rayner 1995).

At the end of the evaluation flight, the fitness of the individual was determined by two separate criterions, based on D and P respectively: the maximal value attained by D during the $10 \mathrm{sec}$ which quantified how far from its reference horizontal path the UAV's trajectory diverged -, and the mean absorbed $\mathrm{P}$ - which measured the mechanical power cost of the achieved flapping movement, given the individual's size and shape:

$$
\text { Fitness }=[-\max (D) ;-\operatorname{mean}(P)]
$$

Both fitness parameters were minus signed, because selection in the Evolutionary Algorithm we used favors high fitness values, and because we aimed at reducing both the trajectory divergence and the power consumption.

The Evolutionary Algorithm we used (epsilon-MOEA; Deb et al. 2005) is a multi-objective 
algorithm, that takes into account the two just-mentioned fitness criterions simultaneously without merging them into one single fitness value as many other algorithms proceed. As an outcome, not a single but several individuals are considered as the "bests". The selection scheme is based on the concept of "domination": within the population, the best individuals are those which have fitness values such that no other individual has higher values on both fitness criterions. The individual is then declared "non-dominated" (see fig.3). By this rule, many individuals in the population can be non-dominated, representing locally optimal compromises between fitness criterions. These individuals are called "Pareto-optimal" solutions, and constitute the most favoured individuals for offspring production. In epsilon-MOEA, Pareto-optimal individuals are placed in what is called an "elite" group, from which one of the two parents implied in each offspring production is systematically chosen at random. The other parent is chosen among the population (see Deb et al. 2005 for details). An important particularity of epsilon-MOEA, compared to some other multiobjective algorithms, is that individual in the elite must differ from each other by some fitness increment: $0.1 \mathrm{~m}$ in trajectory divergence and $1.0 \mathrm{~W}$ in power consumption, in the present case. In other words, the "Pareto front" of the population is interval-sampled. It prevents too much similarity between favoured genitor individuals within the elite, which often causes the premature convergence of evolution toward a local optimum.

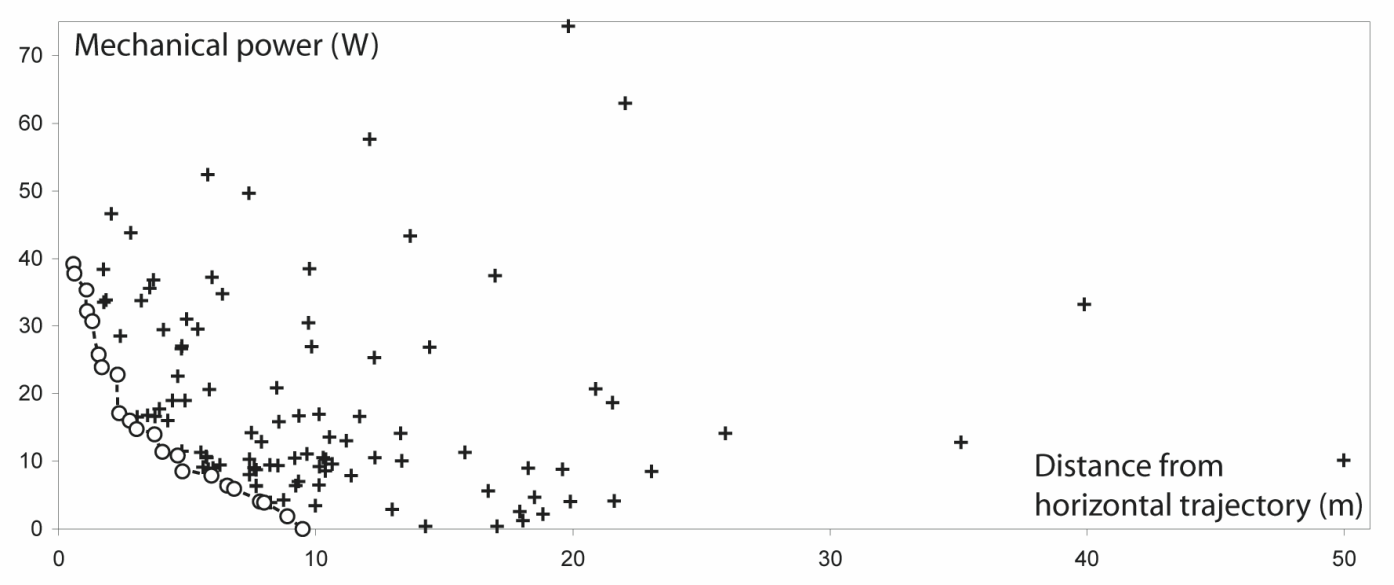

Figure 3: Population and fitness values. Plot of the two fitness criterions (absolute values) for the whole population, at an intermediary step of evolution (example of a $10 \mathrm{~m} / \mathrm{s}$ run after 10000 generation). Each cross represents an individual, i.e. the phenotypic performance of a given genome (a combination of 12 parameter values). The "best" individuals (i.e. "non-dominated individuals" or "Pareto front", see text) are those in the bottom left corner (i.e. lower power consumption and lower departure from reference trajectory), represented as white dots. The next generation will consist of crossing the genome of one individual of the elite (white dot) with one individual of the remaining population (cross). If the offspring is better than both its parents, it will appear closer to the bottom left corner, hence making the Pareto front progress towards better performance.

The sequence of an evolutionary run was as follows:

$1-2000$ individuals with randomly generated genomes were created and individually tested. 2 - the best individuals, in the sense of the multi-objective fitness just exposed, were retained to constitute a first elite group, and the 100 next individuals, only dominated by the elite, constituted the root population for evolution. Other individuals were discarded.

3 - an individual of the elite was randomly chosen for mating with another individual drawn from the population.

4 - the two genomes were crossed to produce an offspring genome: each of the 12 parameters in the offspring genome was randomly chosen from one or the other parent.

5 - the offspring genome was mutated, with a probability of $30 \%$ for each parameter: the corresponding value was randomly increased or decreased by some amount. This amount was randomly drawn from a normal distribution of mean 0 and variance $4 \%$ of the parameter authorized 
range.

6 - the offspring genome was expressed into a phenotype tested into the flight simulator, and its fitness values were measured.

7 - If its fitness values made the offspring a non-dominated individual compared to individuals currently in the population and the elite, it joined the elite group. Otherwise, it was only placed in the "regular" population, with the condition that it was able to replace a relatively worse individual. Otherwise, the offspring was discarded.

8 - steps 3 to 7 were repeated 50000 times. In the following, we will refer to such a cycle as a generation.

At the end of the evolutionary run, the performance, as well as the morphological and kinematic parameters, of individuals in the final elite group were scrutinized.

In order to assess the influence of the flight speed on the evolutionary adaptation of wing size, shape, and movement, we conducted separate evolutionary runs with $6,8,10,12,16$ and $20 \mathrm{~m} . \mathrm{s}^{-1}$ initial horizontal speeds. In all runs, only the flight speed was changed: all individuals had a $500 \mathrm{~g}$ body mass, and the same possible range of variation for other parameters. As AE is a stochastic optimisation method implying many random draws in initial genome generation, as well as in the crossover and mutation processes, we felt necessary to launch 4 duplicate runs per flight speed value in order to estimate how much the resulting adapted morphologies and kinematics converged (or diverged towards different local optima). Hence a total of 24 independent evolutionary runs was conducted initially, each one representing 52000 test flights. As a whole, this represented a total of 3500 virtual flight hours, and approximately 1500 hours of computation on standard personal computers ( $2 \mathrm{Ghz}$ processor with $512 \mathrm{Mb}$ of RAM). In a second stage, some supplementary runs were launched for further analysis (see results and discussion). 


\section{Results and Discussion}

\subsection{Progressive emergence of forward horizontal flight}

Figure 4 shows the progression of the Pareto front ("elite") of the population through successive generations for one of the evolutionary runs aiming at a horizontal flight speed of $10 \mathrm{~m} . \mathrm{s}^{-1}$. The randomly generated front of the initial population already contained different compromise solutions to the horizontal FWF problem. On the right-hand part of the front were located individuals consuming little or no power, but sensibly departing from the horizontal trajectory. These individuals consumed no power because they usually did not move their wings at all, or only through passive movements, i.e. caused by external forces only, and they may be assimilated to "gliders". As a consequence, they systematically lose height during the test flight, as quantified by their score on the distance to the reference trajectory criterion: 15-20 m departure from the horizontal $10 \mathrm{~m} . \mathrm{s}^{-1}$ trajectory at the end of the $10 \mathrm{~s}$. flight. On the left-hand part of the initial front were individuals flying closer to an horizontal path (4 m departure), but at the cost of high power consumption, up to $200 \mathrm{~W}$ of mechanical power in that run. Such high power values were usually due to large wing flapping, at high frequency and with non-optimal incidence angles. Between these two extreme solutions were a few other non-dominated individuals with intermediate performances: better than pure gliders on trajectory departure, and better than the most active flappers on power consumption. After the first 500 generations, the Pareto front progressed significantly, meaning that some offspring were better than their parents and replaced them in the elite group. This was true for all types of solutions: gliders lose less height $(12 \mathrm{~m})$ and active flappers consumed less power (120w). Better intermediate solutions were also found. Similarly, after 1000 offspring generations, the front progressed further, especially for intermediate solutions that became more numerous. Later in the evolution, after 10000 generations, the best glider attained a height loss reduced around $10 \mathrm{~m}$, hence a glide ratio of approximately 10 , since the reference trajectory for $10 \mathrm{~s}$ at $10 \mathrm{~m} \cdot \mathrm{s}^{-1}$ was a 100 $\mathrm{m}$ horizontal path. On the other hand, the departure from horizontal trajectory was reduced to $0.6 \mathrm{~m}$, for $40 \mathrm{~W}$ consumed. During the last 40000 offspring generations, the evolutionary algorithm processed much slower, with the generation of individuals decreasing the power consumption on the "active flapper", on the left-hand side of the Pareto front (mechanical power finally dropped to $25 \mathrm{~W}$ ), and little or no progress on the "glider", on the right-hand side of the front, attaining limits of the airfoil's lift/drag ratio.

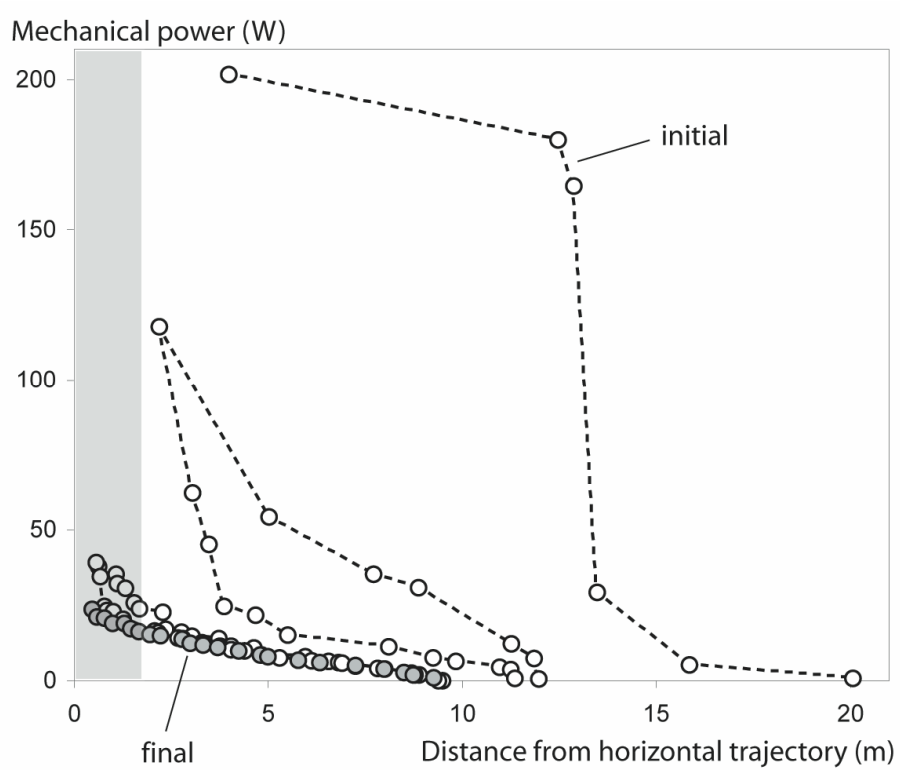

Figure 4: Progression of the elite population through $\mathbf{5 0 0 0 0}$ generations. For the same evolutionary run as in fig. 3 
$(10 \mathrm{~m} / \mathrm{s}$ flight), the Pareto front of the population is plotted at 0 (initial random individuals), 500, 1000, 10000, 20000 and 50000 generations. Within the final elite group (gray dots), only individuals departing less than $2 \mathrm{~m}$ from perfect horizontal flight (gray area) are retained for further analysis.

Evolutionary runs at other reference flight speeds $\left(6,8,12,16\right.$ and $\left.20 \mathrm{~m} . \mathrm{s}^{-1}\right)$ displayed the same trends, though with varying power consumption values. There was an early emergence of glider solutions, which satisfied only one of the fitness criterions, and a more progressive evolution of active flappers and intermediate solutions toward lower power consumption and lower departure from horizontal flight.

At the end of each evolutionary run, after 50000 generations, we retained a few relevant individuals for further analysis of their morphological and kinematic parameters. We were only interested in horizontal flight, and not in glider optimisation, despite the fact that evolution possibly used gliders as parents of active flappers, taking advantage of the multi-objective optimisation scheme.

Therefore, we tolerated a maximal departure of $2 \mathrm{~m}$ from horizontal flight over the 10s flight (see fig. 4). Only individuals satisfying this a posteriori constraint were analysed later in the study. Whether this relative tolerance on trajectory departure represents a significant bias on power consumption can be evaluated by considering that, in the worst case, a $2 \mathrm{~m}$ height loss for a $1 \mathrm{~kg}$ UAV represents $20 \mathrm{~J}$ of lost potential energy during $10 \mathrm{~s}$, hence a power saving of the order of magnitude of a few Watts, which remains tolerable compared to the optimised power levels attained at varying flight speeds (see par. 3.2.1). Furthermore, small errors on altitude might also be due to the lack of pitch closed-loop control, a situation that implies a very accurate parameter tuning that could disappear in a closed-loop control system. All evolutionary runs, at all tested flight speeds, yielded individuals satisfying this constraint after 50000 generations, though in variable number: fewer individuals succeeded in performing a sub-horizontal flight at the lowest $\left(6 \mathrm{~m} . \mathrm{s}^{-1}\right)$ and highest $\left(20 \mathrm{~m} . \mathrm{s}^{-1}\right)$ flight speed, compared to results obtained with intermediate speeds.

\subsection{Morpho-kinematic adaptation to varying flight speeds : comparative analysis}

Figures 5 presents the results of the 24 evolutionary runs we launched, i.e. 4 duplicate runs for 6 flight speeds. We plotted the performance and parameters of the horizontal active flappers at the end of evolution. As an outcome of the stochastic optimisation process, variability between duplicate runs for the same flight speed existed, by varying amounts, depending on the variable considered. However, for most variables, there was a significant convergence between duplicate runs when compared to differences between runs at different flight speeds. In other words, the variability was low enough to identify and discuss comparative adaptations:

\subsection{1. - Power consumption (fig. 5a, b)}

The lowest mechanical power for horizontal FWF of our 500g-bodied UAV, plus the mass of wings and tail (which depended on individual morphology), approximated $15 \mathrm{~W}$, and was achieved at intermediate flight speeds $\left(10,12 \mathrm{~m} . \mathrm{s}^{-1}\right)$. At high speeds, i.e., 16 and $20 \mathrm{~m} . \mathrm{s}^{-1}$, the minimal power to sustain flight increased to almost 20 and $30 \mathrm{~W}$, respectively. On the other hand, at low speeds, the minimal power attained by the most efficient individuals reached much higher values of $60 \mathrm{~W}$ at 8 $\mathrm{m} . \mathrm{s}^{-1}$ and $400 \mathrm{~W}$ at $6 \mathrm{~m} . \mathrm{s}^{-1}$. These results suggest a general U-shaped curve for power consumption across flight speeds, which is consistent with biological models and measurements of FWF power (Rayner, 1999). However, it should be noted that the curves in fig.5a are not directly comparable to biological power/speed curves, because biologists estimate power consumption at varying speeds for a same species, for which only kinematics vary, through the flapping behaviour of a given bird. On the other hand, we present results of (evolutionary) adaptation of both the morphology and kinematics to a given flight speed. Beyond the general U-shape trend, comparisons of absolute power values with biological literature is tempting but somewhat hazardous, as our power values depend on many model parameters that are inevitably only partly bio-mimetic, for example airfoil characteristics. Moreover, empirical measurements of mechanical power in bird species have been conducted on smaller bird species, because of wind tunnel size constraints. With these restrictions 
in mind, we still can refer to the results of Dial et al. (1997), who measured a minimum 9 W.kg-1 power (per kilogram of bird mass) in the magpie (Pica pica). Tobalske et al. (2003) measured a minimum of $17 \mathrm{~W} \cdot \mathrm{kg}^{-1}$ in the cockatiel (Nymphicus hollandicus), and $31 \mathrm{~W} \cdot \mathrm{kg}^{-1}$ in the ringedturtled dove (Streptopelia risoria). Our minimal value of $20 \mathrm{~W} . \mathrm{kg}^{-1}$ after mass correction (fig. 5b) falls among those biological values. However, maximal measured mechanical power consumption is $54 \mathrm{~W} . \mathrm{kg}^{-1}$ in the dove (Tobalske et al. 2003), a value that many of our optimised individuals exceed by a large amount, especially at low speed. Though the mass-specific power limit in birds is probably above the above-mentioned values, as $80 \mathrm{~W} \cdot \mathrm{kg}^{-1}$ have been documented in some species during take-off (Askew et al. 2001), it is clear that the power values we obtain at $6 \mathrm{~m} . \mathrm{s}^{-1}$ are unrealistic at $400 \mathrm{~W}$ or $500 \mathrm{~W} \cdot \mathrm{kg}^{-1}$. Technological considerations suggest that the mechanical power produced on a real UAV prototype would hardly exceed $200 \mathrm{~W}$. This rather optimistic figure is obtained assuming a single, $150 \mathrm{~g}$ state-of-the-art electric motor (e.g. ModelMotors 2820/8), absorbing $400 \mathrm{~W}$ of electric power, and a global 50\% efficiency for the whole flapping mechanism. Two main hypotheses can explain the unreasonable energetic levels we obtain for slow flapping flight :

(i) Our UAV's DOFs are too restrictive compared to those of real birds, and constrain the possible kinematics so much that slow flight cannot be performed efficiently.

Our flight mechanics model does not take unsteady aerodynamics into account, and thus our candidate individuals cannot use effects such as delayed stall to increase airfoil performance (Vogel 1994).

Since we obtain extreme power values only at low flight speed, and since unsteady aerodynamics are known to grow in relative importance at low flight speed, the second hypothesis is theoretically well grounded. As for the first hypothesis, it will be discussed later during the analysis of morphological and kinematic parameters (par. 3.2.5).

\subsection{2. - Wing area $\left(a_{w}\right)$ adaptation (fig. $\left.5 c, d\right)$}

The optimal wing area emerging from evolution depended greatly on the flight speed: the general trend was that $\mathrm{a}_{\mathrm{w}}$ decreased with an increasing flight speed. As the mass of the UAV's body remained $0.5 \mathrm{~kg}$, the adaptation of wing area implied an increase of wing loading for higher flight speeds, a well known relationship for all flying objects, as there is a physical proportionality relationship between the natural flight speed and the square root of wing loading (e.g. Norberg 1990). At 6-8 m.s $\mathrm{s}^{-1}$, the mean $\mathrm{a}_{\mathrm{w}}$ was near $0.3 \mathrm{~m}^{2}$. At 10 and $12 \mathrm{~m} . \mathrm{s}^{-1}, \mathrm{a}_{\mathrm{w}}$ decreased to approx. 0.2 and $0.15 \mathrm{~m}^{2}$ respectively, i.e., to values approaching the natural wing areas of $500 \mathrm{~g}$-bodied birds such as Marsh-Harrier, Shearwater or Hooded Crow (Greenewalt 1962). At 16 and 20 m.s ${ }^{-1}$, evolution converged to the minimum allowed wing area value of $0.1 \mathrm{~m}^{2}$, indicating that selection strongly favoured highly loaded individuals at these high speeds, mimicking a "duck-like" adaptation. It should be noted that optimal individuals at the end of our evolutionary runs are necessarily "specialists" of the flight speed at which they were selected, which is different in natural bird species, whose characters (e.g. wing loading) probably result from selective compromises over the whole flight speed range they practice. Whether wing areas values selected here at a given flight speed would remain functional at other flight speeds, by changing the wing movement only, is an issue dealt with later (par. 3.5).

\subsection{3. - Wing aspect ratio $\left(\lambda_{\mathrm{w}}\right)$ adaptation (fig.5e, f)}

Evolution yielded high aspect ratio values at almost all tested flight speeds. The maximal ratio value of 10 was reached by most individuals at $6,8,10,12$ and $16 \mathrm{~m} . \mathrm{s}^{-1}$. It is only at $20 \mathrm{~m} . \mathrm{s}^{-1}$ that an optimal aspect ratio averaging 8.5 was obtained. A $\lambda_{w}$ lower than 7 was never retained at the end of the evolutionary runs. High aspect ratios have the beneficial effect of decreasing the induced drag (eq.14), and hence the forward thrust force that must be generated by flapping. However, for a given wing area, wings with a high aspect ratio have a lower chord and thus experience lower Reynolds number, which increases the airfoil friction drag (eq.15). Optimal aspect ratio should theoretically result from a compromise between these contradictory effects. The optimal value 
therefore depends on the flight speed, the wing area, and on the sensibility of the airfoil performance to Reynolds number. Moreover, in FWF, aspect ratio has other implications: more power will be needed to accelerate/decelerate a high aspect ratio wing, which has higher inertia around the shoulder joint. On the other hand, the flapping frequency would possibly be reduced with a wing with a high aspect ratio, as wing tip velocity induced by flapping will be increased by higher wingspans, thus generating higher thrust forces. In the present case, with the specific characteristics of our UAV in terms of mass, area, airfoil and flight speeds, it appears that the optimal $\lambda_{\mathrm{w}}$ is around 10 or more, which is above values observed for similarly-sized birds (7.7 on average; Norberg 1990). This difference has two main possible origins:

(i) our simulator did not take structural resistance into account, whereas a bird's fitness strongly depends on maintaining the integrity of its wing structure with a reasonable safety factor. In other words, depending on the material used, it is possible that high aspect ratio wings would bend or break during flapping at a high frequency. This is a first selective pressure towards low aspect ratio wings that is lacking in our study.

(ii) most importantly, we only selected our UAVs for forward flight. Thus no selective pressure was placed on manoeuvrability, on flight in obstructed areas (vegetation), or simply on wing folding for walks on the ground, which are factors that all favour the selection of lower aspect ratios in birds, at the expense of a slightly lower aerodynamic efficiency (Norberg 1990, 2002). Considering these limitations compared to natural conditions, it is not surprising that simulated evolution converged towards what can be considered as high aspect ratio "open space flyers", somewhat analogous to marine bird species that are almost $100 \%$ occupied at flying, like albatrosses and other Diomedeidae or Procellaridae.

3.2.4. - Flapping frequency (f) and Dihedral amplitude (amp $\left.{ }_{\mathrm{di}}\right)$ adaptation (fig. $5 \mathrm{~g}, \mathrm{~h}$ )

At intermediate speeds ( 10 and $12 \mathrm{~m}^{-1} \mathrm{~s}^{-1}$ ), the flapping stroke frequency was about $3 \mathrm{~Hz}$. This value is in the lower biological range $(2-10 \mathrm{~Hz}$ at this mass, Norberg 1990), which is not surprising given the long, seabird-like wings of our UAV: with this morphology, sufficient thrust can be generated with a low flapping frequency (Norberg, 1990, p.177). As a point of comparison, the Kelp gull (Larus dominicanus) has a "natural" flapping frequency of $3.5 \mathrm{~Hz}$ (Pennycuick 1996), for mass and area $\left(0.89 \mathrm{~kg}\right.$ and $\left.0.23 \mathrm{~m}^{2}\right)$ characteristics comparable to those of our intermediate speed UAV. However, this species has a slightly lower aspect ratio of approximately 7.5.

Slow and fast flight both implied higher frequency values (closer to $5 \mathrm{~Hz}$ on average), which contribute to explain the observed increase in power consumption at those speeds. Concerning the stroke amplitude, there was a less clear adaptive trend, with most individuals presenting a dihedral amplitude in the $25-45^{\circ}$ range, with some increase at the lowest and highest flight speeds, contributing further to the increase in mechanical power. As a whole it seems that variations in both the stroke frequency and amplitude were implied in the adaptation of flapping kinematics to flight speed. However, both variables did not vary similarly: for example, only frequency increased from 12 to $16 \mathrm{~m} . \mathrm{s}^{-1}$ (with a slight decrease in stroke amplitude), whereas only amplitude increased from 16 to $20 \mathrm{~m} . \mathrm{s}^{-1}$, suggesting that these variables exhibit a rather complex adaptive landscape. These trends are unfortunately not easily comparable to intraspecific biological kinematic data because, in the present case, the wing area varied between flight speeds. Nevertheless, it should be mentioned that biological data show that flapping frequency depends on flight speed in some species, while it remains fairly constant in others (Tobalske and Dial 1996, Park et al. 2001).

3.2.5. - Sweep amplitude $\left(\mathrm{amp}_{\mathrm{sw}}\right)$ and offset (off $\left.\mathrm{sw}\right)$ adaptation (fig. $\left.5 \mathrm{i}, \mathrm{j}\right)$

The potential usefulness - or worthlessness - of an articulated wing in a FWF UAV is an interesting issue that has not been directly addressed previously. Of course birds and bats have elbow and wrist and use these DOFs in flight, with an amount depending on species and flight speed (Tobalske and Dial 1996, Park et al. 2001, Tobalske et al. 2003). From an adaptationist, functionalist point of view, this suggests that an articulated wing may be aerodynamically useful. On the other hand, 
other factors constrain the presence of wing articulations in birds and bats : first of all, the heredity of a vertebrate limb organisation plan is an historical, contingent constraint that questions the purely functional necessity of an articulated wing. For example, insect can fly efficiently without articulated wings. Moreover, an articulated wing may be beneficial to other functional aspects than forward flight, e.g., to increased manoeuvrability, and the simple biological necessity to fold wings on the ground represent a potentially strong natural selective pressure that is not necessarily relevant for an UAV. Hence it was interesting to test whether the presence of a wrist in our simulated wing was used by evolution for purely forward flight, and to quantify its possible beneficial effects. Figure 5i shows that the sweep of the outer panel was used by almost all optimised individuals, at all flight speeds. Between 8 and $20 \mathrm{~m} . \mathrm{s}^{-1}$, the amplitude of the sweep did not vary much in a consistent manner, and averaged $25^{\circ}$. A different pattern appeared at $6 \mathrm{~m} . \mathrm{s}^{-1}$, as most individuals at this lowest speed used a much higher amount of sweep, attaining 60 to $80^{\circ}$. Fig $5 \mathrm{j}$ shows how the sweep was synchronised with the dihedral : the sweep (SW) tended to have a $0-25 \%$ period offset compared to the dihedral (DI) which, according to equations (1) and (2), shows that a maximal sweep angle (i.e. adducted wing tips, minimal wingspan) was attained in the second half of the upstroke, whereas a zero sweep angle (i.e. fully extended wing) was attained in the second half of the downstroke. This is close to what is observed in birds, for which it has been usually reported that the maximal wingspan occurs at mid-downstroke, and minimal wingspan at mid-upstroke, which corresponds to a $25 \%$ value for off $\mathrm{sw}_{\mathrm{sw}}$. The amount of wing retraction was globally less than in real birds: given our UAV morphology (fig. 1), the ratio of minimal to maximal wingspan was 0.95 for $25^{\circ}$ of sweep, 0.75 for $60^{\circ}$ and 0.58 for $80^{\circ}$. Birds for which this same "span ratio" variable has been measured in flight exhibit much lower values, usually below 0.5 (Tobalske and Dial 1996, Park et al. 2001, Tobalske et al. 2003). Moreover, most of these species (e.g. Barn Swallow [Hirundo rustica], Pigeon [Columba livia], Cockatiel [Nymphicus hollandicus]) have a tendency to more retract their wings at a higher speed, which is not observed in the present case. As already noted, this discrepancy can partly result from the fact that our UAV's size changed between flight speeds, hence the wing area adaptation need not be achieved through partial wing folding as in real birds. It is also important to note that the bird species thus investigated are far from the "seabird" morphotype, for which data on span ratio is lacking. We speculate from personal observations that wing adduction in gulls and akin species is less pronounced than in pigeons for example. There are also structural reasons why our UAV retracts its wings rather modestly compared to birds. First, the wrist in our UAV cannot be adducted, as only the wingtips can. This constraint de facto limits the span ratio to a minimal value of 0.5 . Second, as wrists cannot move forward or backward relative to the body, we considered the possibility that a strong sweep of the external panel would separate the lift center of the wing from the center of gravity the UAV, and hence cause pitch torques and instability issues. However, this hypothesis is partly refuted by the fact that individuals at $6 \mathrm{~m} . \mathrm{s}^{-1}$ succeed in using up to $83^{\circ}$ of sweep. It remains that the limitations we put on wing retraction, suggested by anticipated constraints on prototype construction, might indeed partly cause the very high power consumptions we obtained at low speed. It could indeed prevent our UAV from exploring some of the wing movements a bird can achieve, which are especially refined at low speeds, as illustrated and discussed in the biological literature mentioned herein. Although the wing retraction possibilities of our UAV were modest compared to those of birds and bats, it remains that the wrist sweep was almost systematically used, thus suggesting that it allowed the generation of more efficient aerodynamic forces. To test this idea further, we quantified the power gained from wrist movements (see par. 3.4).

3.2.6. - Shoulder and wrist incidence rotations (fig. 5k, 1, m, n, o, p)

The shoulder incidence position (SINC) determines the angle between IP and body, whereas the wrist incidence position (WINC) determines the angle between OP and IP. Hence the angle between OP and body results from the sum of SINC and WINC. Plots of reference angles versus flight speed ( ref $_{\text {sinc }}$, ref $_{\text {winc }}$, fig. $5 \mathrm{k}$ and 5l) show that IP tended to have a higher angle of incidence at low speed, but not OP: negative angles at the wrist tended to compensate the positive angles at the shoulder. 
Concerning the variation of the angle of incidence, its amplitude ( $a m p_{s i n c}$, amp $p_{w i n c}$, fig. $5 \mathrm{~m}, 5 \mathrm{n}$ ) tended to increase with lower flight speeds, for both IP and OP, which is in agreement with kinematic data in birds which associate higher variations in the wing angle of attack with slow flights (Heddrick et al. 2002). It is noteworthy that SINC and WINC values being relative angles between body and wing panels, they provide only an indirect information on the aerodynamic angle of attack, which depends on the incident air speed induced by the flapping stroke, and on possible changes in the body tilt angle (see par. 3.2.7). The offset between the incidence and dihedral

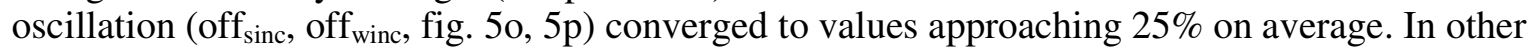
words, a maximal positive incidence was attained at mid-upstroke, while a minimal incidence (often negative angles between body and wing panels) was attained at mid-downstroke. As a direct outcome, this pattern tend to maintain the wing airfoil at low angles of attack throughout the stroke, thus maximizing the lift/drag ratio (fig. 2). 

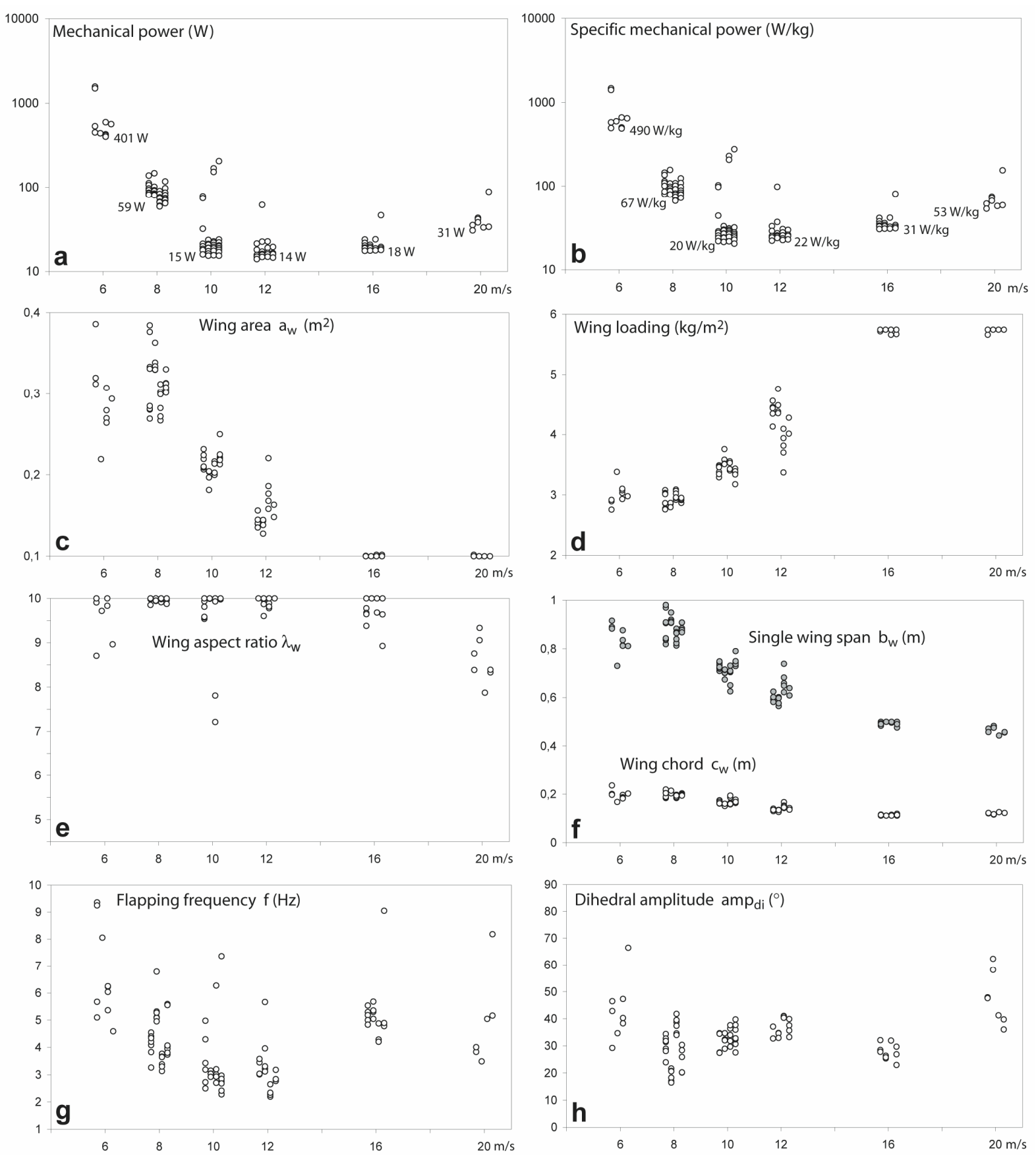

Figure 5: Results of morpho-kinematic adaptation to varying flight speed. The characters of the best individuals resulting from 4 duplicate evolutionary runs at each flight speed $(6,8,10,12,16$ and $20 \mathrm{~m} / \mathrm{s}$ ) are plotted (individuals generated during the same run are aligned vertically). For all genomic parameters, the vertical range of the graph equals the authorised search space during evolution. 

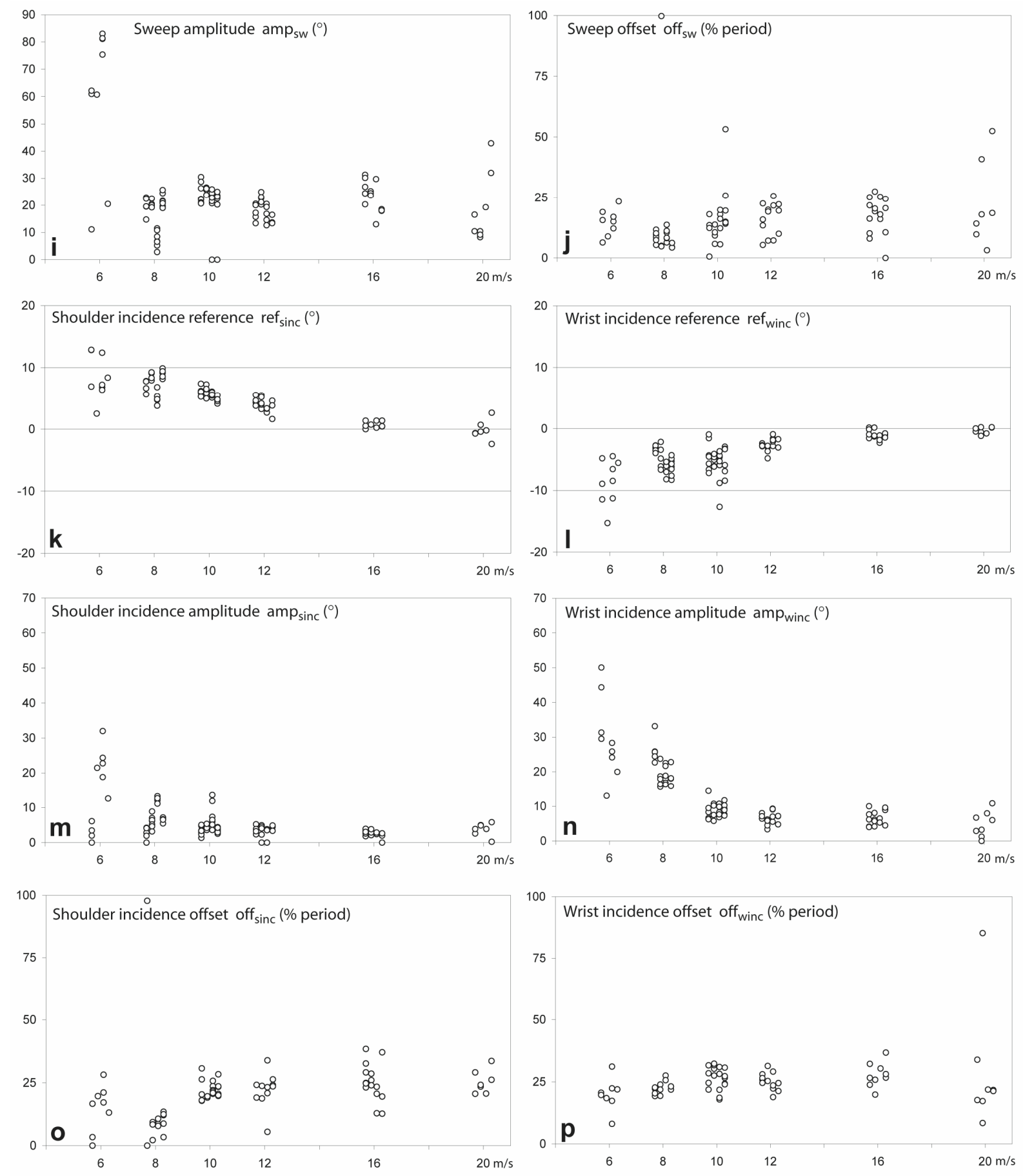

Figure 5 (continued). 
3.2.7. - Analysis of aerodynamic forces in representative individuals.

To investigate the respective aerodynamic role of inner/outer panels during downstroke and upstroke throughout the flight speed range, we plotted the aerodynamic forces generated by IP and OP along the flight path for one individual. We chose the most power-saving individual at each flight speed. As a consequence of the dual-objective optimisation scheme, these individuals do not necessarily have the best performances in terms of horizontality of flight. On the contrary, turns out that the best individuals in terms of flight horizontality achieved their flight at the expense of power consumptions that were an order of magnitude higher, with much more variability between duplicate runs, than those of the six individuals selected here - clearly suggesting that they were much less aerodynamically efficient, and hence less interesting and representative of aerodynamic optimisation. As a complementary illustration, we also produced in-flight motion videos of these six individuals (available on http://animatlab.lip6.fr). Numerical values of all parameters for these individuals are presented in table 1.

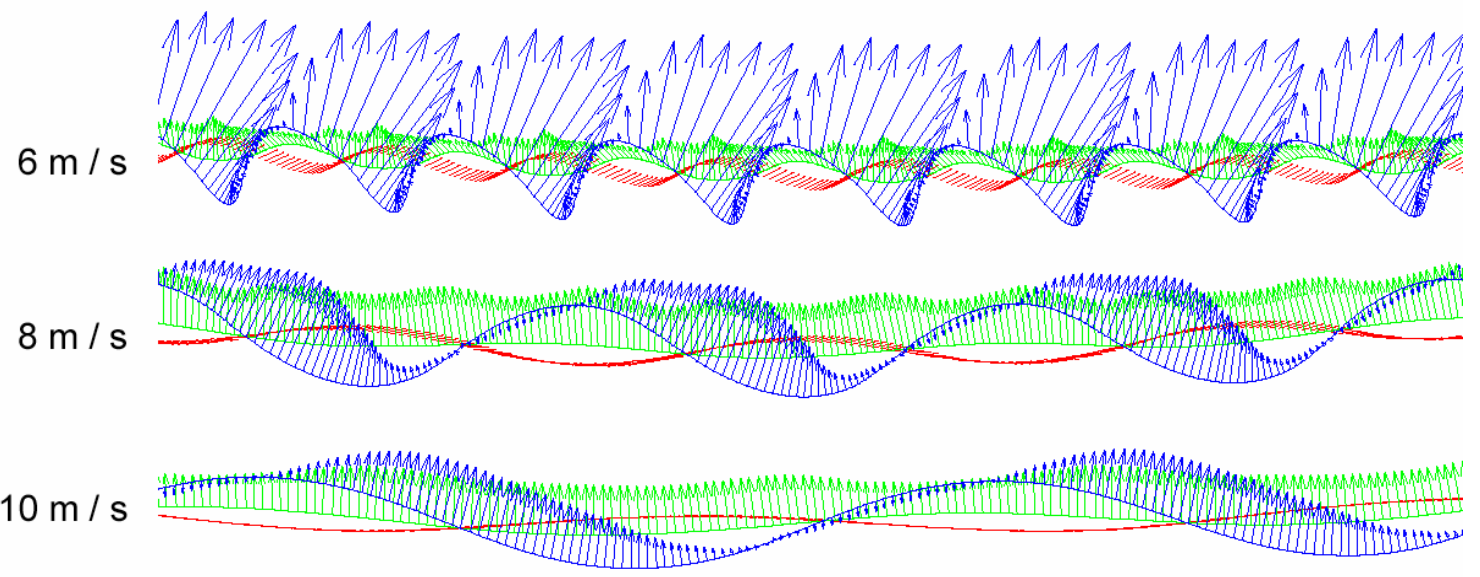

$12 \mathrm{~m} / \mathrm{s}$

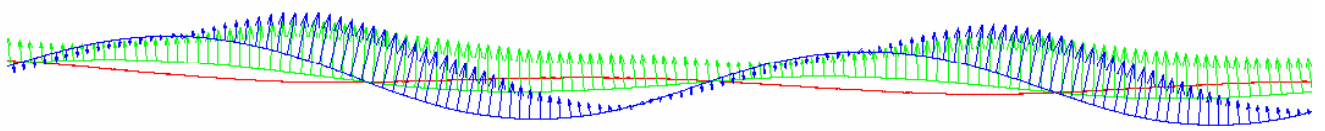

$16 \mathrm{~m} / \mathrm{s}$
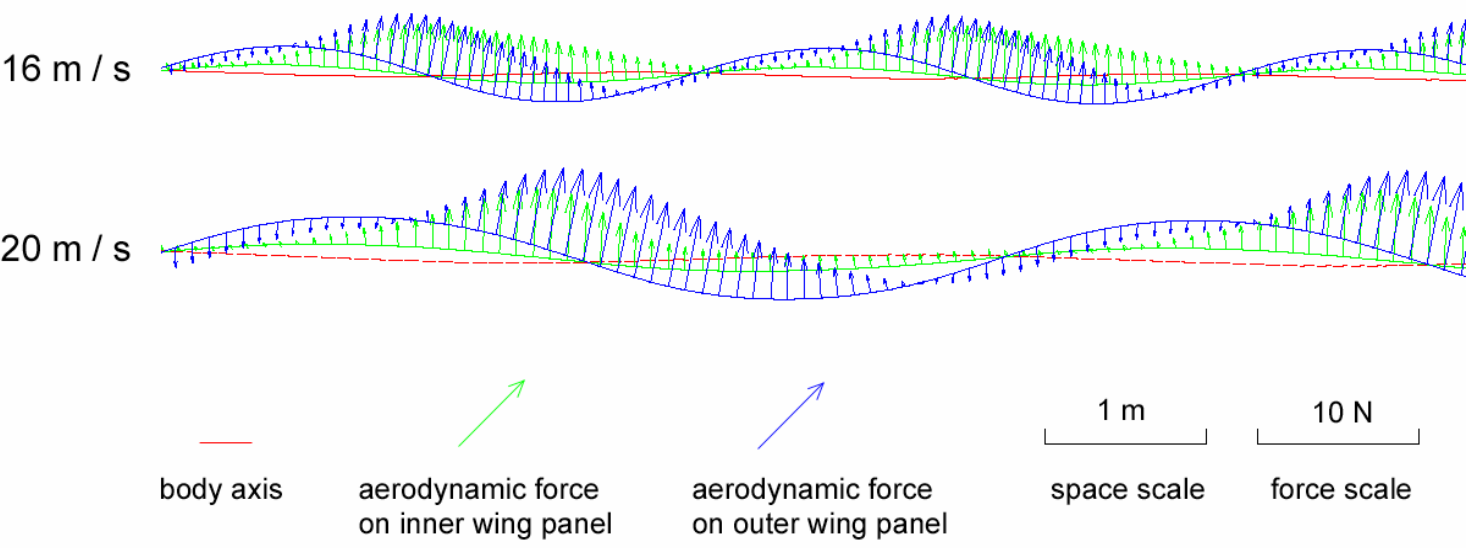

Figure 6: Aerodynamic forces on the wing panels of six optimised UAVs. The force vectors are summed over the "wing elements" (WELs, see "Flight Simulation" section) constituting a single (inner or outer) panel. The vectors' origins are on a line representing the trajectory of each panel's center of area. Tail and fuse forces are not represented for clarity. 


\begin{tabular}{|c|c|c|c|c|c|c|}
\hline Flight speed (m/s) & 6 & 8 & 10 & 12 & 16 & 20 \\
\hline \multicolumn{7}{|l|}{ Fitness } \\
\hline mean $\mathrm{P}(\mathrm{W})$ & 401 & 59 & 15 & 14 & 18 & 31 \\
\hline $\max D(m)$ & 1.11 & 1.95 & 1.98 & 1.77 & 1.80 & 1.48 \\
\hline \multicolumn{7}{|l|}{ Genome } \\
\hline$a_{w}\left(m^{2}\right)$ & 0.264 & 0.299 & 0.225 & 0.145 & 0.100 & 0.100 \\
\hline$\lambda_{w}$ & 10.0 & 10.0 & 10.0 & 10.0 & 9.6 & 8.4 \\
\hline$f(H z)$ & 6.25 & 3.30 & 2.41 & 3.03 & 4.83 & 4.01 \\
\hline$a m p_{d i}\left({ }^{\circ}\right)$ & 40.3 & 34.0 & 32.8 & 32.7 & 27.9 & 47.7 \\
\hline$a m p_{s w}\left({ }^{\circ}\right)$ & 75.4 & 10.8 & 25.0 & 20.0 & 30.2 & 16.6 \\
\hline off $_{\mathrm{sw}}(\%)$ & 17.0 & 11.2 & 14.6 & 13.5 & 25.1 & 9.7 \\
\hline $\operatorname{ref}_{\text {sinc }}\left({ }^{\circ}\right)$ & 6.4 & 4.8 & 4.8 & 4.6 & 1.4 & -0.7 \\
\hline $\operatorname{amp}_{\operatorname{sinc}}\left({ }^{\circ}\right)$ & 18.8 & 11.1 & 4.3 & 3.2 & 3.1 & 3.9 \\
\hline off $_{\operatorname{sinc}}(\%)$ & 21.1 & 7.8 & 20.0 & 24.1 & 32.6 & 20.6 \\
\hline $\operatorname{ref}_{\text {winc }}\left({ }^{\circ}\right)$ & -11.3 & -5.4 & -3.3 & -2.8 & -0.9 & 0.0 \\
\hline $\operatorname{amp}_{\text {winc }}\left({ }^{\circ}\right)$ & 24.2 & 16.3 & 7.3 & 7.1 & 6.2 & 6.7 \\
\hline off $_{\text {winc }}(\%)$ & 22.3 & 25.6 & 27.2 & 24.5 & 23.7 & 33.9 \\
\hline \multicolumn{7}{|l|}{ Other information } \\
\hline$b_{w}(m)$ & 0.81 & 0.87 & 0.75 & 0.60 & 0.49 & 0.46 \\
\hline $\mathrm{c}_{\mathrm{w}}(\mathrm{m})$ & 0.18 & 0.19 & 0.17 & 0.13 & 0.11 & 0.12 \\
\hline$r_{b}(m)$ & 0.05 & 0.05 & 0.05 & 0.04 & 0.03 & 0.03 \\
\hline total span (m) & 1.68 & 1.78 & 1.55 & 1.24 & 1.01 & 0.95 \\
\hline $\mathrm{m}_{\mathrm{b}}(\mathrm{kg})$ & 0.500 & 0.500 & 0.500 & 0.500 & 0.500 & 0.500 \\
\hline $\mathrm{m}_{\mathrm{w}}(\mathrm{kg})$ & 0.272 & 0.327 & 0.213 & 0.110 & 0.063 & 0.063 \\
\hline $\mathrm{m}_{\mathrm{t}}(\mathrm{kg})$ & 0.048 & 0.058 & 0.038 & 0.020 & 0.011 & 0.011 \\
\hline total mass $(\mathrm{kg})$ & 0.820 & 0.885 & 0.751 & 0.630 & 0.574 & 0.574 \\
\hline wing loading $\left(\mathrm{kg} / \mathrm{m}^{2}\right)$ & 3.1 & 3.0 & 3.3 & 4.3 & 5.7 & 5.7 \\
\hline specific power (w/kg) & 490 & 67 & 20 & 22 & 31 & 53 \\
\hline
\end{tabular}

Table 1: Parameters of the most power-saving individual at each flight speed.

A first remark concerns the trajectory and position of the body. It followed an oscillating path, ascending during downstroke and descending during downstroke. More interestingly, the body axis took a significantly tilted position at low speed (up to approx. $30^{\circ}$ at $6 \mathrm{~m} . \mathrm{s}^{-1}$ ), a tendency observed and measured with comparable amounts in birds (Tobalske and Dial 1996, Tobalske et al. 2003). Considering the forces generated by OP it appeared that the force generation was almost fully concentrated during the downstroke, regardless of the flight speed. These downstroke OP forces had both vertical (upward) and horizontal (forward) components, showing that OP had both a lifting and a propulsive function. At the beginning of the upstroke, weak lifting forces were also produced, but shifted to weak downward forces later in the upstroke. As a whole, OP was almost inactive during the upstroke at all flight speeds. Interestingly, at $6 \mathrm{~m} . \mathrm{s}^{-1}$, this asymmetric OP force pattern between downstroke and upstroke was achieved through a drastic variation in the relative airspeed : the simultaneous effects of a high wrist sweep - causing backward retraction of the outer wing part during upstroke - and the body tilt angle produced an almost zero OP horizontal speed during upstroke, while the same panel was greatly accelerated during downstroke. At higher flight speed, 
such a velocity difference was not observed with comparable amounts, thus suggesting that the absence of OP upstroke forces was mainly caused by placing the OP airfoil at a non-lifting angle of attack.

Concerning IP, the force generation exhibited a different pattern. Forces were more evenly distributed among down- and upstroke, and included a lift (upward) and a drag (backward) component. This showed that IP, contrary to OP, did not usually participate to the propulsion of the UAV. This IP force pattern changed somewhat at the highest speeds, with a weakening of upstroke forces, with relatively stronger forces generated during the downstroke, and with a slight forward component of the generated force at mid-downstroke. As a whole, this IP force pattern at high speed was closer to the previously described OP force pattern.

The comparison of the mean force amplitude over the full stroke on OP and IP showed a clear OP domination at $6 \mathrm{~m} . \mathrm{s}^{-1}$, and a more even repartition over the wing span at other speeds. This is explained by the fact that the airflow is dominated by wing flapping at slow flight speeds (Hedrick et al. 2002), and hence depends on the distance from the articulated dihedral joint.

Concerning the down-/upstroke repartition for the wing as a whole, more lifting forces and all propulsive forces were generated at the downstroke, at all flight speeds. This was mainly due to OP generating forces during downstroke only. However, according to what has been described above, this downstroke domination tend to be less obvious at intermediate speeds $\left(8-12 \mathrm{~m} . \mathrm{s}^{-1}\right)$ as lift produced by IP during upstroke took relatively higher importance. This is globally convergent with the results of Hedrick et al. (2002) in the Dove and Cockatiel: these birds appear to have a more continuous lift generation at intermediate speeds (Hedrick et al. 2002, Tobalske et al. 2003). These significant variations in force generation modes across the flight speed range suggest that the limited kinematics of our UAV compared to real birds, which prevent very adducted upstrokes (e.g. "feathered" upstroke, Tobalske and Dial 1996), still leave room for efficient adaptation in the aerodynamic flight regime.

\subsection{Flight stability and robustness of the "open-loop" kinematic control}

Aside from pure energetic performance, the applicability of our optimised flapping flight kinematics to a real UAV prototype depends on its ability to generate a stable flight. Our UAV's wing movement control was truly "open loop" in the present work, i.e. the UAV had no information on its flight variables and was not able to change its kinematics to correct its flight trajectory. For this reason, we anticipated that the UAV's trajectory, even after kinematic optimisation, would necessarily diverge from horizontal flight after a short time, presumably because of pitch instability. Therefore, we a priori considered the optimised kinematics produced here as basic wing motion laws that would necessitate a supplementary, higher level "closed-loop" controller to achieve flight stability in our UAV, like the ones described in Mouret et al. (submitted).

To verify this presumption, we extended to 60 seconds the flying time of the optimised individuals (table 1), instead of the $10 \mathrm{~s}$. of regular evaluation flight, in order to study the type of instability that might thus occur. Results are presented as flight trajectories in fig.7. Surprisingly, the longitudinal (pitch axis) stability was much better than expected, as any strong vertical trajectory divergence after the first 10 s of evaluation time was never observed. Instead, despite the symmetric flight kinematics, we observed some lateral instability, i.e. the UAV progressively engaged into a descending spiral turn. This spiral occurred after a longer time at high flight speed, individuals flying at 16 and $20 \mathrm{~m} . \mathrm{s}^{-1}$ being able to fly for $60 \mathrm{~s}$. without being affected. Even during these relatively longer forward flights, pitch stability was observed throughout, the sub-horizontal trajectory being maintained for the whole minute (i.e. 920 and $1200 \mathrm{~m}$ distances, respectively). Moreover, a few supplementary flight tests, with variable initial speeds on the same individual, demonstrated that some individuals were able to passively correct large flight speeds discrepancies. For example, the individual optimised for $20 \mathrm{~m} . \mathrm{s}^{-1}$ and launched at $6 \mathrm{~m} . \mathrm{s}^{-1}$ was able to return to its horizontal $20 \mathrm{~m} . \mathrm{s}^{-1}$ flight within a minute (fig.7). Unfortunately, the opposite test of launching the individual optimised for $6 \mathrm{~m} . \mathrm{s}^{-1}$ at $20 \mathrm{~m} \cdot \mathrm{s}^{-1}$ was unsuccessful, as lateral instability soon occurred 
(fig. 7).

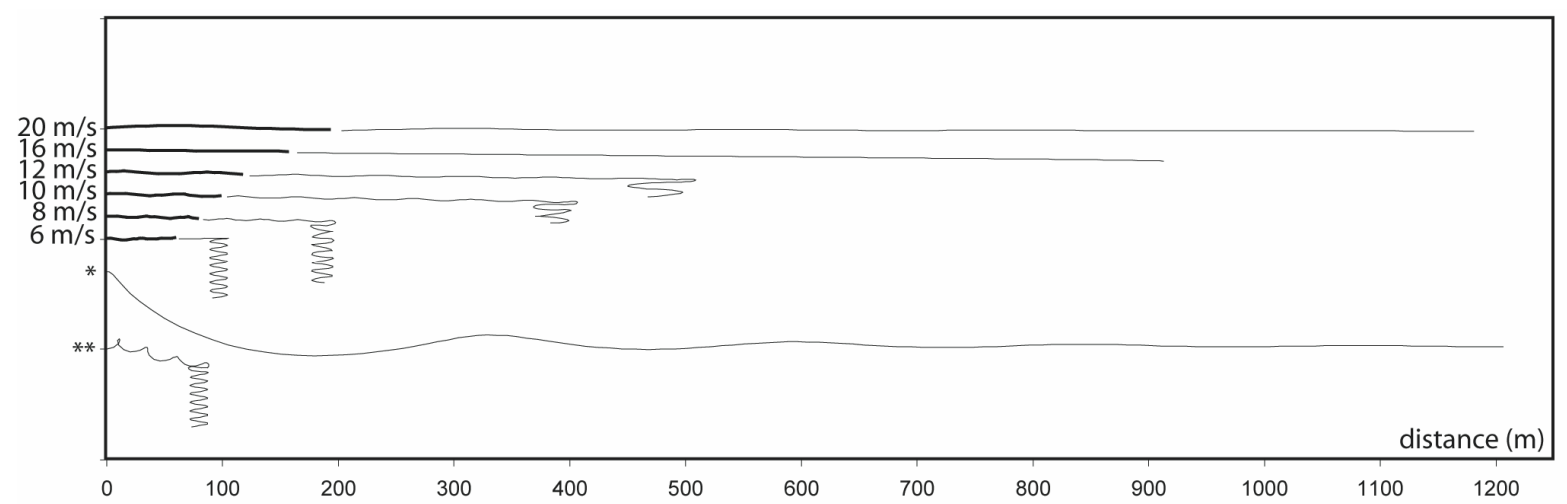

Figure 7: Flight trajectories (side views) of optimised UAVs during extended 60s. flights. Thick lines represent the $10 \mathrm{~s}$. initial evaluation flight. Thin lines represent the $50 \mathrm{~s}$. flight prolongation, and demonstrate good longitudinal stability, but lesser lateral stability at low speed (spiral dive) of open-looped controlled FWF UAVs (see text). * : trajectory of the individual optimised for $20 \mathrm{~m} / \mathrm{s}$, launched at a lower speed $(6 \mathrm{~m} / \mathrm{s})$ : horizontal flight at $20 \mathrm{~m} / \mathrm{s}$ is recovered. ${ }^{* *}$ : trajectory of the individual optimised for $6 \mathrm{~m} / \mathrm{s}$, launched at a higher speed $(20 \mathrm{~m} / \mathrm{s})$ : horizontal flight is not achieved, due to lateral instability.

It is probable that our initial choice of placing the CG at $25 \%$ of wing chord, where lift forces apply, and of providing the morphology with a large tail helped the UAV to achieve pitch stability, at least in gliding flight. However, it remains that most non-optimised flapping kinematics during evolutionary exploration had as a first consequence to destabilise the UAV and to place it on an erratic flight trajectory. In this perspective, it is an interesting result that optimised UAVs were finally able to achieve a reasonable amount of passive pitch stability in flight. This suggests that the necessarily superimposed closed-loop controller mentioned above will eventually have relatively little corrective work to do to provide long-term longitudinal - and lateral - stability.

\subsection{Effect of wrist lock: Usefulness of an articulated wing}

Previously exposed results show that the wrist sweep was used by almost all optimised individuals. At $6 \mathrm{~m} \cdot \mathrm{s}^{-1}$, the sweep was used at its maximum, and force plots (fig. 6) suggest that it allowed to accelerate the wing tip during downstroke. However, the role of a relatively lower amount of sweep at higher speed was less obvious, and thus could appear a priori less important for flight performance. To test further the usefulness of a functional wrist, which would imply a more complex UAV prototype structure, we launched additional evolutionary runs in the same original conditions except that the wrist DOFs were disabled. We launched 2 runs at each flight speed with the wrist sweep locked ( $\mathrm{SW}=0^{\circ}$, i.e. wing fully extended), and 2 more runs at each flight speed with the wrist sweep and the wrist incidence locked ( $\mathrm{SW}=0^{\circ}$ and WINC $=0^{\circ}$, i.e. wing fully extended and same incidence for IP and OP). After 50000 generations for each of these 24 evolutionary runs, we compared the power consumption for the best horizontal flappers, with the same original $2 \mathrm{~m}$ tolerance in the departure from reference trajectory.

Results (fig.8) show that disabling the wrist sweep implied a 8-79\% increase in power consumption, depending on flight speed, except at $8 \mathrm{~m} \cdot \mathrm{s}^{-1}$ where no power cost was observed. This surprising pattern at $8 \mathrm{~m} . \mathrm{s}^{-1}$ is partly explained by the fact that the original individual at $8 \mathrm{~m} \cdot \mathrm{s}^{-1}$ used $11^{\circ}$ of wrist sweep only, which affords the sweep-locked individual the possibility of attaining very similar kinematics. At other flight speeds, where the original sweep amplitude was $17-75^{\circ}$, locking the wrist sweep caused evolution to attain more costly kinematics, even when the original sweep amplitude was modest and its aerodynamic role unclear. For example, a 79\% power increase at 12 $\mathrm{m} . \mathrm{s}^{-1}$ resulted from preventing the original $25^{\circ}$ sweep amplitude. The sweep's beneficial effect tend to be less important at high speed. Globally, these results suggest that a functional wrist is really useful even in pure forward flight, at most flight speeds. Locking both the wrist sweep and the 
incidence rotations caused dramatic power increases at most speeds (10-252\%). Stressing further the crucial role of an articulated wing for FWF, this finding demonstrates that much power can be saved by allowing different incidence angles between rigid wing panels. Such variation in the incidence angle along the wing span is well known in birds and bats (Norberg 1990, p.118), though achieved through a flexible wing surface composed of feathers or membrane respectively, rather than through articulated rigid panels. It seems likely that we would get lower power consumption if we allowed a continuous twisting of our UAV wing, but this possibility generates additional technical complications if one wants to maintain an efficient airfoil (e.g. DeLaurier 1999).

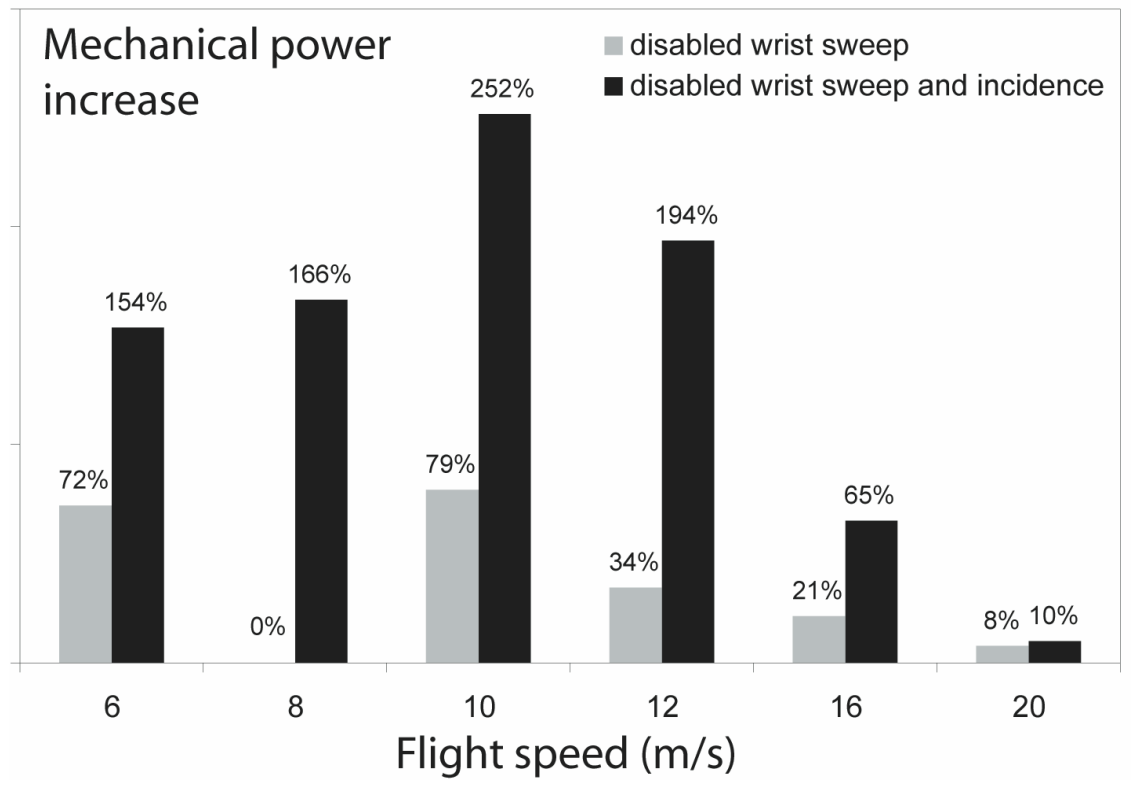

Figure 8: Effect of wrist lock on power consumption.

\section{$\underline{\text { 3.5. Evolution at fixed size and shape : Readaptation of kinematics to different flight speeds }}$}

As already explained, evolving the wing size, the shape and the kinematics at a given flight speed probably tends to produce "specialist" individuals, i.e. whose characters are well optimised for the considered flight speed, but non optimal at any other regime. To evaluate the degree of specialisation of the optimised individuals we previously obtained, we let the kinematics of some individuals evolve again at different flight speeds, while keeping the initial morphology (i.e. wing area and aspect ratio) unchanged. We performed 12 additional evolutionary runs: the morphology of the most power-saving individual at $6 \mathrm{~m} . \mathrm{s}^{-1}(\mathrm{~A})$ was used to re-evolve the kinematics at 10 and 16 $\mathrm{m} . \mathrm{s}^{-1}$ (2 duplicate runs per flight speed). Similarly, the best morphology adapted for $10 \mathrm{~m} . \mathrm{s}^{-1}$ (B) was tested at 6 and $16 \mathrm{~m} . \mathrm{s}^{-1}$, while new kinematics for the best morphology at $16 \mathrm{~m} . \mathrm{s}^{-1}$ (C) were evolved at 6 and $10 \mathrm{~m} \cdot \mathrm{s}^{-1}$. Results in terms of minimal power consumption are presented in fig. 9 . A first finding was that each of the three morphologies remained the most power-saving solutions at their original flight speeds, which comforted the idea that separately evolving the kinematics on a constrained morphology has indeed a cost, as compared with the original choice of simultaneously evolving the wing morphology and kinematics. However, the performances of B at $6 \mathrm{~m} . \mathrm{s}^{-1}$ and A at $10 \mathrm{~m} . \mathrm{s}^{-1}$ were only a few watts above the original values. This is not surprising given that $\mathrm{A}$ and $\mathrm{B}$ had rather close wing area values $(0.264$ and $0.225 \mathrm{~m} 2$ respectively) and hence similar wing loadings (3.10 and $3.34 \mathrm{~kg} . \mathrm{m}-2$ ). Evolving the kinematics for A or B at $16 \mathrm{~m} . \mathrm{s}^{-1}$, as compared to the high-speed specialist (C, $0.1 \mathrm{~m} 2$ wing area), had a more obvious consequence since necessary power was approximately doubled. Aerodynamically, this cost is caused by the high drag forces exerted on a large wing at a high speed, as compared to a smaller wing. Biologically, this same energetic cost explains why gulls do not fly forward as fast as ducks. Most interesting was the fact 
that $\mathrm{C}$ was able to fly at $10 \mathrm{~m} \cdot \mathrm{s}^{-1}$ with a 5-fold increased cost compared to $\mathrm{B}$, and simply unable to fly at $6 \mathrm{~m} . \mathrm{s}^{-1}$ : evolution failed to find any kinematics generating an horizontal flight. The best individual in the two runs lost $8 \mathrm{~m}$ of height in $10 \mathrm{~s}$. This result expresses the difficulty to generate much lift with a small wing, and can be biologically illustrated by the tendency of ducks and similar species to refrain from flying slowly - though they can occasionally do it. As a whole, these tests showed that all individuals resulting from our optimisation runs had not attained the same degree of specialisation. Namely, whereas morphologies evolved initially for low to medium speed flight could adapt their kinematics to fly faster, the opposite was not true : high speed morphs could loose the ability to fly at lower flight speeds. Technically, this suggests that one would better choose A or B morphologies for a FWF UAV prototype rather than C, if the versatility in terms of flight speed range is an objective.

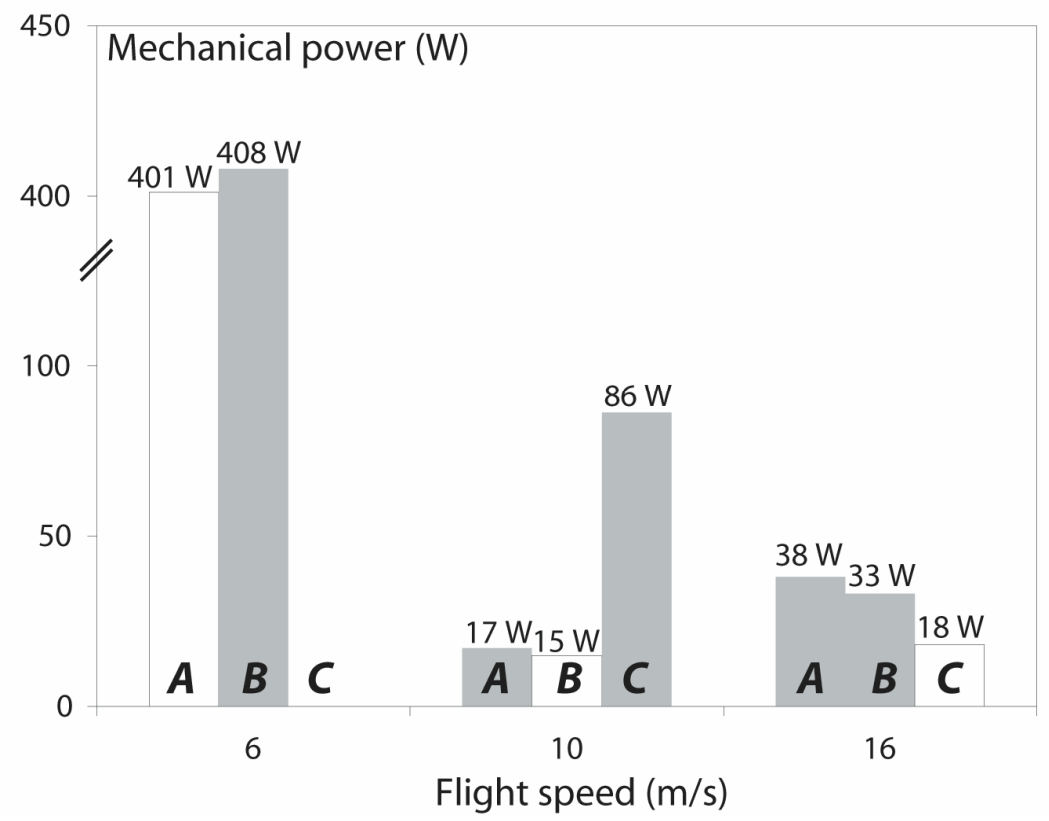

Figure 9: Re-adaptation of kinematics to different flight speeds. Three individual morphologies (A, B, C), initially optimised for different flight speed $(6,10$ and $16 \mathrm{~m} / \mathrm{s}$ respectively, white bars), were used to re-evolve new kinematics (without changing the morphology) at other speeds (gray bars). No experiment converged with the $\mathrm{C}$ morphology at $6 \mathrm{~m} / \mathrm{s}$. See text for analysis of the consequences on power consumption. 


\section{Conclusions and perspectives}

The proximal aim of the present work was to find optimal morphologies and kinematics for a flapping, articulated-wing mini-UAV. By using Artificial Evolution on a bird-like parameterised morphology, we were able to find morphological parameters and flapping stroke kinematics that achieve forward flight at medium speed $\left(10-12 \mathrm{~m}_{\mathrm{s}}^{-1}\right)$ for an estimated mechanical cost comparable to that of real birds: approximately $15 \mathrm{~W}$ or $20 \mathrm{~W} \cdot \mathrm{kg}^{-1}$. These parameters correspond to a $0.2 \mathrm{~m}^{2}$, high aspect ratio wing, flapping around $3 \mathrm{~Hz}$, looking like a gull in many aspects. Even more indicative is the fact that evolution also yielded angular variation laws for each articulation of this UAV (shoulder and wrist) capable of producing efficient lift and thrust forces throughout the flapping stroke with the chosen wing airfoil.

In a short-term perspective, we plan to test this morphology and its associated kinematics on a real UAV prototype, secured on a robotic arm (research in progress in the ROBUR project). We expect of course that real energy levels will differ somewhat from simulation predictions, and that some fine-tuning of kinematics will be necessary to correct for the unescapable simulation approximations.

Beyond medium-speed steady flight, the main interest of flapping wing flight for an UAV is the potential to vary its flight speed to a large amount, and to achieve special flight modes such as hovering or gliding. Though we could not cover the whole flight domain extensively in the present study, we already gained valuable information on the energetic consequences of speed variation. Flying at high speed $\left(16-20 \mathrm{~m} . \mathrm{s}^{-1}\right)$ could be achieved at rather low cost $(20-30 \mathrm{~W})$ in a small UAV $\left(0.1 \mathrm{~m}^{2}\right)$, but with a loss of flight abilities at lower speeds. On the other hand, we observed that it was possible to re-adapt the kinematics of a larger UAV $\left(0.2-0.3 \mathrm{~m}^{2}\right)$, initially optimised for lower speed flight, to fly at high speed for a reasonable power cost $\left(30-40 \mathrm{~W}\right.$ at $\left.16 \mathrm{~m} . \mathrm{s}^{-1}\right)$. This second strategy hence seems more promising in the perspective of a versatile real UAV.

Flying at low speed $\left(6-8 \mathrm{~m} . \mathrm{s}^{-1}\right)$ appeared very costly, and unrealistic power consumption were attained at $6 \mathrm{~m} \cdot \mathrm{s}^{-1}$. Though the steady aerodynamics that were used in our simulation probably underestimated the lift production in slow flight, it is possible that our articulated morphology, as it stands, is inadequate for very slow or hovering flights, at least for a $1 \mathrm{~kg}$ UAV prototype. Even birds of this size hardly achieve hovering for more than a few seconds. At another end of the flight domain, the fact that our UAV was able to achieve gliding flight with a decent sinking speed (around $-1 \mathrm{~m} . \mathrm{s}^{-1}$ ) is encouraging and suggests that bio-mimetic energy-saving behaviours, through soaring in ascending air, are within reach of our UAV's abilities.

Another original result brought by this work concerns the usefulness of an articulated wing for flapping flight: even without considering turning or manoeuvrability issues, we already learned that the movement of the wrist may have a strong influence on energy saving in forward flight, especially at medium-speed flight: compared to monolithic wings, allowing sweep and incidence rotations of no more than $20-25^{\circ}$ at mid-span can drastically reduce the power consumption of a FWF UAV, provided that these supplementary movements are well synchronised with the flapping stroke. In other words, this finding suggests that implementing an articulated wing on a UAV prototype might be worth the implied technical complications.

Finally, from a methodological point of view, we mainly relied on biological data to validate/analyse our results. This was facilitated by the fact that we initially constrained the evolutionary search space to bio-mimetic morphologies and kinematics, in agreement with the rationale of the animat approach. Although more efficient flapping-wing machines may exist outside these boundaries, we are convinced that discovering and analysing such non-biomimetic FWF solutions would probably have been much more difficult, as we could not have relied on 
zoological records as an helpful documented referential for a preliminary validation of our results before experiments on real prototypes. Conversely, some of our simulation results should be useful to biologists. Although being not as reliable as real-world measures, Artificial Evolution coupled with environment simulation may be considered as a very valuable, fully controlled experimental framework serving to test the effect of all sorts of constraints - physical, historical or developmental - on the course and outcome of adaptation of all sorts of biological characters. Such an evolutionary modelling approach should be especially useful for functional morphologists and biomechanicists (de Margerie et al. 2005). As illustrated by the present work on FWF, many experiments that would be impossible in a wind tunnel may be relatively easily reproduced in simulation, and yield to original results, such as:

- "flapping wing flight without the brain": our flight experiments with purely open-looped kinematics demonstrate that flapping a wing efficiently does not necessarily compromise the passive gliding-flight stability, nor does a short forward flight necessarily require any stabilizing neural control. This kind of result, possibly reinforced by experiments on variable tail areas for example, could provide useful information on the degree of active stabilisation the extant birds need to achieve in flight, and also produce valuable arguments on the morphological and neurological prerequisites for the emergence of flight in vertebrates.

- "flapping wing flight with a stiff wing": we were able to independently lock some degrees of freedom in our morphology, which is hardly conceivable on a real bird in a wind tunnel, and to evaluate the consequences on the energetic cost of flapping flight after letting the UAV readapt its kinematics. It was thus demonstrated that even a limited sweep movement of the outer wing part can save a significant part of power consumption, and that the ability to vary the incidence angle along wing span is a crucial feature of FWF at this size.

We are convinced that, as well as roboticists may draw helpful inspiration from zoological records and Darwinian evolution (Meyer and Guillot, in press), biologists interested in adaptation at a high integration level, such as organismal biologists or ecologists, can find new lines of evidence by using Artificial Evolution on modelled organisms. Although the structure of our study - notably the type of Artificial Evolution experiments that were conducted and the way the results were analysed - was primarily aimed at helping designing an UAV, and not at yielding biologically relevant findings, we hope the present work still participates at demonstrating these reciprocating interests.

\section{Acknowledgement}

The authors thank T. Druot for helpfull advice on using and improving the FMFAW flight simulator. This study was partly funded by the post-doctoral fellowship of the french "Direction Generale de l'Armement" (DGA) for E. de Margerie. 


\section{Reference}

Angeli A, Filliat D, Doncieux S and Meyer J-A 2006. 2D simultaneous localization and mapping for micro aerial vehicles. In Proceedings of the European Micro Aerial Vehicles (EMAV 2006) conference.

Askew G N, Marsh R L and Ellington C P 2001. The mechanical power output of the flight muscles of blue-breasted quail (Coturnix chinensis) during take-off. J. Exp. Biol. 204: 3601-3619.

Barate R, Doncieux S, and Meyer J-A 2006. Design of a bio-inspired controller for dynamic soaring in a simulated UAV. Bioinspiration \& Biomimetics, 1:76-88.

de Margerie E, Tafforeau $P$ and Rakotomanana L 2006. In silico evolution of functional morphology: a test on bone tissue biomechanics. J. R. Soc. Interface 3: 679-687.

Deb K, Mohan M and Mishra S 2005. Evaluating the epsilon-Domination Based Multi-Objective Evolutionary Algorithm for a Quick Computation of Pareto-Optimal Solutions. Evolutionary Computation 13: 501-525.

DeLaurier J 1999. The development and testing of a full-scale piloted ornithopter. Canadian Aeronautics and Space Journal 45: 72-82.

Dial K P, Biewener A A, Tobalske B W and Warrick D R 1997. Mechanical power output of bird flight. Nature 390: 67-70.

Doncieux S, Mouret J, Muratet L, and Meyer J-A 2004. The ROBUR project: towards an autonomous flapping-wing animat. In Proceedings of the Journées MicroDrones, Toulouse.

Druot $\mathrm{T}$ 2004. Technical report on the implementation and validation of a flight mechanics simulator for flapping articulated wings. Available on http://animatlab.lip6.fr

Goldberg D E 1989. Genetic Algorithms in Search, Optimization, and Machine Learning. AddisonWesley, Reading, Mass.

Greenewalt C H 1962. Dimensional relationships for flying animals. Smithson. Misc. Collect. 144: $1-46$.

Greenewalt C H 1975. The flight of birds. Trans. American Philosophical Society 65: 1-67.

Hedrick T L, Tobalske B W and Biewener A A 2002. Estimates of circulation and gait change based on three-dimensional kinematic analysis of flight in cockatiels (Nymphicus hollandicus) and ringed turtle-doves (Streptopelia risoria). J. Exp. Biol. 205: 1389-1409.

Meyer J-A and Guillot A In press. Biologically-inspired robots. In Siciliano B and Khatib O editors, Handbook of Robotics. Springer-Verlag.

Mouret J-B, Doncieux S, Druot T and Meyer J-A (submitted). Evolution of closed-loop neurocontrollers for flapping-wing animats.

Muratet L, Doncieux S, Brière Y and Meyer J-A 2005. A contribution to vision-based autonomous helicopter flight in urban environments. Robotics and Autonomous Systems, 50(4):195-209. 
Norberg U M 1990. Vertebrate flight. Springer, Berlin.

Norberg U M 2002. Structure, form and function of flight in engineering and the living world. $J$. Morphol. 252: 52-81.

Park K J, Rosen M and Hedenström A 2001. Flight kinematics of the barn swallow (Hirundo rustica) over a wide range of speeds in a wind tunnel. J. Exp. Biol. 204: 2741-2750.

Pennycuick C J 1996. Wingbeat frequency of birds in steady cruising flight: New data and improved predictions. J. Exp. Biol. 199: 1613-1618.

Rakotomamonjy T, Le Moing T and Ouladsine M 2004. Simulation model of a flapping wing micro air vehicle. EMAV 2004 Conference, Braunschweig, Germany.

Rayner J M V 1999. Estimating power curves of flying vertebrates. J. Exp. Biol. 202: 3449-3461.

Salles R and Schiele A 2004. Analysis of flapping-wing robots for planetary exploration. An evolutionary approach. Proceedings of the $8^{\text {th }}$ ESA Workshop on Advanced Space Technologies for Robotics and Automation ASTRA 2004.

Selig M 1997. Summary of low speed airfoil data - Vol. 2. SoarTech Aero Publications, Virginia Beach, VA, USA. www.ae.uiuc.edu/m-selig/uiuc_lsat.html

Shim S Y, Kim S J and Kim CH 2004. Evolving flying creatures with path-following behaviour. Proceedings of the Ninth International Conference on the Simulation and Synthesis of Living Systems (Alife IX).

Smith R 2006. Open Dynamics Engine - www.ode.org

Tobalske B W and Dial K P 1996. Flight kinematics of blacked-billed magpies and pigeons over a wide range of speeds. J. Exp. Biol. 199: 263-280.

Tobalske B W, Hedrick T L, Dial K P and Biewener A A 2003. Comparative power curves in bird flight. Nature 421: 363-366.

van Breugel F and Lipson H 2005. Evolving buildable flapping ornithopters. GECCO 2005.

Van den Berg C and Rayner J M V 1995. The moment of inertia of bird wings and the inertial power requirement for flapping flight. J. Exp. Biol. 198: 1655-1664.

Vogel S 1994. Life in moving fluids. Princeton, NJ: Princeton University Press.

Withers P C 1981. An aerodynamic analysis of bird wings as fixed airfoils. J. Exp. Biol. 90: 143162. 